\title{
Information transfer and recovery for the sense of touch
}

\author{
Chao Huang ${ }^{1,2^{*}}$, Bernhard Englitz ${ }^{1 *}$, Andrey Reznik ${ }^{2}$, Fleur Zeldenrust ${ }^{1}$, Tansu Celikel ${ }^{1}$ \\ (1) Department of Neurophysiology, Donders Institute for Brain, Cognition and Behaviour, \\ Radboud University, Nijmegen - the Netherlands \\ (2) Laboratory of Neural Circuits and Plasticity, \\ University of Southern California, Los Angeles - CA, USA \\ * Denotes equal contribution \\ Correspondence should be addressed to celikel@neurophysiology.nl
}

\section{Abstract}

12 Transformation of postsynaptic potentials (PSPs) into action potentials (APs) is the rate-limiting step of

13 communication in neural networks. The efficiency of this intracellular information transfer also powerfully

14 shapes stimulus representations in sensory cortices. Using whole-cell recordings and information-theoretic

15 measures, we show herein that somatic PSPs accurately represent stimulus location on a trial-by-trial basis

16 in single neurons even 4 synapses away from the sensory periphery in the whisker system. This information

17 is largely lost during AP generation but can be rapidly ( $<20 \mathrm{~ms}$ ) recovered using complementary

18 information in local populations in a cell-type-specific manner. These results show that as sensory

19 information is transferred from one neural locus to another, the circuits reconstruct the stimulus with high

20 fidelity so that sensory representations of single neurons faithfully represent the stimulus in the periphery,

21 but only in their PSPs, resulting in lossless information processing for the sense of touch in the primary

22 somatosensory cortex. 


\section{Introduction}

25 Neural information processing requires signal transformation every time the information is

26 transferred from one neuron to another. This transformation is performed in postsynaptic neurons by

27 integrating spatiotemporally distributed synaptic inputs and generating action potentials, which then

28 propagate information across synaptically coupled neurons. For each processing step, how much

29 information is retained, how much of it is transferred to a postsynaptic neuron, how much is lost, and

30 whether local networks can fully recover the lost information during this intracellular sub-to-suprathreshold

31 information transfer are questions that have yet to be answered. In an accompanying paper (Zeldenrust et

32 al., 2020) we show on a single neuron level that how much information is lost during action potential

33 generation depends on the cell-class. On a network level, the effectiveness of this input-to-spike operation

34 depends on the connectivity, the code used between the sender (i.e. presynaptic neurons), and the receiver

35 (i.e. postsynaptic neurons) as well as the noise characteristics of the channel. Since many of these are

36 currently impossible to assess experimentally, the rules of information transfer in biological circuits, with

37 the exception of cell-type-specific intracellular information transfer in single neurons as outlined in the

38 accompanying article (Zeldenrust et al., 2020), are still largely unknown.

39 Sensory systems in particular offer unique opportunities to study information processing in neural

40 circuits. If the primary function of a sensory circuit is to faithfully and reliably represent the environment

41 (Azarfar et al., 2018; DeCharms and Zador, 2000; Diamond et al., 1999; Knudsen et al., 1987), a substantial

42 part of the sensory information in the periphery should be represented throughout the sensory circuits in the

43 form of neural signals. Sensory systems are commonly organized in the form of topographical maps, where

44 sensory receptors in the periphery are represented by topographically organized groups of neurons along

45 the sensory axis (Harding-Forrester and Feldman, 2018; Kole et al., 2018; Petersen, 2019). However, the

46 functional role of these topographical maps for sensory processing is still not clear (Chklovskii and

47 Koulakov, 2004; Diamond et al., 1999; Kaas, 1997; Weinberg, 1997). Understanding the mechanisms of 
48 information processing, transfer and recovery is particularly important in sensory circuits, as the efficacy

49 of signal transformation should determine the extent, speed, and accuracy of sensory representations.

50 Stimulating single neurons in the sensory and motor cortices can result in observable behavioral

51 responses such as whisker movement (Brecht et al., 2004; Doron et al., 2014; Houweling and Brecht, 2008;

52 Voigts et al., 2008). However, single neurons carry surprisingly little information in the rate and timing of

53 their action potentials (Alenda et al., 2010; Panzeri et al., 2001; Petersen et al., 2001; Quian Quiroga and

54 Panzeri, 2009). Given that pooling information across simultaneously recorded neighboring neurons

55 minimally contributes to the information carried in local populations because neighboring neurons carry

56 largely redundant information (Petersen et al., 2002, 2001), the target postsynaptic neurons are likely to

57 reconstruct the stimulus by spatiotemporal integration across behaviorally relevant spatial and temporal

58 scales (Azarfar et al., 2018; Celikel and Sakmann, 2007).

Here, we performed intracellular recordings and used computational modeling to address the

60 principles of information processing in the somatosensory cortex. Surprisingly (to us), we found that the

61 sensory stimulus can be fully reconstructed with the information available in the subthreshold responses of

62 single excitatory neurons (i.e. the recorded EPSPs in L2/3 neurons). Up to $90 \%$ of this information is lost

63 during intracellular information transfer, i.e. when an action potential is generated from these subthreshold

64 responses, in agreement with previous observations on the information content of action potentials in barrel

65 cortical neurons (Alenda et al., 2010; Panzeri et al., 2001; Petersen et al., 2002). In vivo information loss is

66 likely to exceed this value, due to background ongoing activity (Destexhe et al., 2003). Next, we assessed

67 information recovery on the population level using an analysis based on bootstrapped groups of neurons

68 recorded in vitro. We found that information lost during action potential generation can be fully recovered

69 by as little as 100 neurons with a time resolution of 2-3 ms. Finally, we turned to a realistic and well-

70 constrained simulation of a barrel column (Huang et al., 2020) to study the relation between encoding

71 strategies in L4 and decoding strategies in L2/3 to determine the mechanisms of information recovery. 
72 Comparing candidate encoding strategies in the L4 population, we found that a population rate code (using

73 peri-stimulus time histograms obtained in the in vivo recordings) is unsuitable for information transfer in a

74 cortical network because the trial-to-trial reliability is too low to fit the high information recovery that we

75 found as in our experiments. Codes with higher trial-to-trial reliability in timing and rate perform

76 substantially better, with optimal performance reached if neurons fire reliably across trials. In this case, the

77 L4 activity can be fully decoded by small groups ( 25 cells) of both excitatory and inhibitory neurons in

78 L2/3 within $\sim 20 \mathrm{~ms}$ after stimulus onset, and within a few $\mathrm{ms}$ after the first spike response. In summary,

79 we show that intracellular information transfer is highly lossy, and thus potentially selective. However, by

80 combining the limited but complementary information in the spike trains of L2/3 inhibitory and excitatory

81 neurons, single neurons could fully reconstruct stimulus resulting in lossless representation of sensory

82 information in their subthreshold responses. 


\section{Results}

\section{$84 \quad$ L2/3 single cell responses to in vivo whisker stimulation}

85 We performed whole-cell current-clamp recordings of Layer (L) 2/3 pyramidal neurons in the juvenile rat

86 primary somatosensory cortex, in the barrel cortical subregion under ketamine anesthesia. During these in 87 vivo recordings, sensory stimulation was provided by direct stimulation of the principal and 1st order

88 surround whiskers with a piezo stimulator in 2 directions (up-down: Fig. 1A). The cumulative synaptic 89 input in response to these stimuli was quantified in properties of the somatic post-synaptic potential (PSP), 90 i.e. the onset time, slope, and peak amplitude (Fig. 1B-D). Principal whisker stimulation-evoked PSPs

91 exhibited the shortest latency, as well as the highest slope and amplitude, in comparison to PSPs evoked by 92 the stimulation of surrounding whiskers, in agreement with previous observations (Brecht et al., 2003). 93 Although the PSPs were highly reliable (PW: 99.8\%, SW: 91.8\% of trials evoked PSPs), action potentials

94 (spikes) were sparse and unreliable, even after principal whisker deflections (PW: 6.2\% (SD 8.6\%) ; SW:

$95 \quad 1.7 \%$ (SD 2.9\%) of trials included evoked APs).

\section{Properties of sub- \& suprathreshold responses of $\mathbf{L} 2 / 3$ neurons to $\mathbf{L} 4$ stimulation}

97 Mutual information calculations require long sampling durations, which limits the possibilities for unbiased 98 calculation of information processing with high-dimensional naturalistic stimuli in vivo. Therefore we 99 performed acute slice experiments with simplified stimuli (Fig.1E-P). Bipolar electrodes in L4 were used 100 to deliver square pulses with varying slopes as described before (Fig.1E-F; Huang et al., (2016); see 101 Materials and Methods). Visualized L2/3 neurons were recorded in whole-cell current clamp configuration.

102 PSP responses of L2/3 neurons systematically varied with the four stimulus patterns (Fig. 1G-J). Spike 103 responses showed a similar dependence on the stimulus slope when averaged over multiple trials, with 
104 delayed spike times, increased threshold and decreased spike probability for shallower slopes of stimulation

105 (Fig. 1K-M).

106 The average properties shown above indicate that individual cells qualitatively correspond to

107 whisker deflections mimicking spatial stimuli. However, during sensory processing, animals have to deduce

108 object location from single trials, not from averages over many trials, which is only possible when trial-to-

109 trial variability is low. Spikes exhibited a far greater temporal trial-to-trial variability than PSPs (Fig. 1N-

110 P). PSP onset times showed an average progression with stimulus slope, with a small trial-to-trial

111 variability (Fig. $1 \mathrm{~N}, \mathrm{SD}=0.42-0.63 \mathrm{~ms}$ ), whereas spike time variability was threefold higher (Fig.1O, SD

$112=1.2-2.3 \mathrm{~ms})$. Therefore, spike times could only to a very limited degree be predicted on the basis of PSP

113 onset times, with spikes often occurring with significant and variable delays (Fig. 1P, 2.8-4.7 (SD 1.1-2.1)

$114 \mathrm{~ms})$. Spike generation was also failure prone, with average spike failures rates up to $31.7 \%$ of the trials

115 [Range : 0-85\%]. While spike failures bear information in rate-based codes, in timing-based codes

116 information is missing when the neuron fails to fire an action potential. To study the influence of trial-to-

117 trial variability in timing and rate on stimulus information, we calculated Shannon's mutual information

118 between the stimulus and several PSP and spike properties. This mutual information provides largely

119 agnostic estimates of the transmitted information between stimulus and response.

\section{Information transmission between somatic PSPs and Spikes}

121 How much information does a somatosensory neuron carry about the sensory stimulus (S) and how much

122 of this information does it transfer to its postsynaptic targets? Surprisingly, the information between a single

123 somatic PSP and the stimulus contains the bulk ( 95\%, I(S;PSP) vs. H(S), Fig. 2A) of the entropy of the

124 sensory stimuli. The information between the onset time (81\%, Fig. 2B), slope (8.4\%, Fig. 2B) and

125 amplitude (6.4\%, Fig. 2B) of the PSP and the stimulus contribute largely independently to the total

126 information content of a PSP (Fig. 2C). However most of this information is lost upon spike generation 
127 (down to 24\%, I(S; St,Vt), Fig. 2A), where spike timing (St, 16\%, Fig. 2D) and voltage threshold (Vt, 6.2\%,

128 Fig. 2D) carry most of the stimulus information contained in the spikes.

129 We can directly quantify how much of the information in the PSP is transferred to the spike (see

130 Materials and Methods). Unsurprisingly, the total entropy in the PSP (i.e across onset, slope and amplitude

131 together) exceeds the stimulus information multifold (6.4 bits, 3.2-fold, Fig. 2E). The transferred

132 information from PSP to spike amounts to $22 \%$ and $15 \%$ of the PSP entropy for St and Vt respectively (Fig.

$1332 \mathrm{E}$, comparison of medians). However, most of this information is redundant, since the actual amount of 134 stimulus information contained in a spike is much lower (0.41 bit, Fig. 2A). The individual PSP properties 135 on the other hand contribute slightly synergistically to the timing information in the spike (Fig. 2F, 6.4\%, $\left.136 \mathrm{p}<10^{-5}\right)$. Consequently, while a substantial amount of the information about the stimulus in the PSP is 137 transferred to the spike, this information is insufficient to encode the present stimulus space at the single 138 neuron level on a single neuron and single trial basis.

\section{Information recovery in local neural populations in silico and in vitro}

140 If the PSP-to-spike transformation causes a dramatic drop in information about the stimulus carried in the

141 neural activity, how can the somatic PSPs of L2/3 neurons carry near complete information (Fig.2A)? Since

142 these neurons are four synapses away from the sensory periphery, a recovery of information has to occur at 143 the network level. Information recovery was analyzed both in an anatomically and physiologically well144 constrained network model of a rat barrel column (Fig. 3) and on bootstrapped populations of the in vitro 145 data (Fig. 4, see Materials and Methods and Huang et al., (2020)).

146 The model has anatomically correct numbers and laminar locations of major classes of inhibitory 147 and excitatory neurons in L4 and L2/3 (Fig. 3A), single neuron dynamics based on experimental 148 observations as well as statistically defined connectivity and synaptic transmission parameters. Stimulation 149 was provided analogously to the in vitro stimulation in L4, using previously collected L4 peri-stimulus time 
150 histograms (PSTH) of principal and surround whisker stimulation in vivo (Fig. 3B, L4 response to principal

151 whisker in gray (Celikel et al., 2004). PSTHs of simulated L2/3 excitatory and inhibitory neurons

152 correspond to experimentally observed ones under similar conditions (De Kock et al., (2007), Fig. 3B red

153 and blue, respectively).

154 In the model, the timing of and information in PSPs and spikes closely matched the properties of

155 the real neurons in biological networks (compare Fig 3 to Fig.1-2). In the simulations, the trial-to-trial

156 variability in spike timing was substantially and significantly larger than the variability in PSP timing

157 (Fig. 3C, compare with Fig. 1A). Stimulus information was nearly fully retained in the somatic PSPs of

158 excitatory neurons (Fig. 3D, red, 88.8\% of the stimulus entropy), yet reduced substantially (20.1\%) during

159 spike generation, similar to our observations in biological neurons (Fig. 2A). Interestingly, inhibitory

160 neurons carried significantly less information in their PSPs (Fig. 3D, blue, 77.3\%), but also exhibited less

161 information loss during spike generation (43.6\%).

162 In the bootstrapped data, the stimulus information could be almost fully recovered from populations

163 of excitatory L2/3 neurons recorded in vitro (>81.1\%, Fig. 4B). The amount of information recovered was

164 substantially greater for decoding including timing $(81.1 \%$, in timing of the 1 st spike, binned at $2 \mathrm{~ms}, 100$

165 cells), than for rate based only decoding (50.5\%, red vs. light red) and was largely independent of the

166 population size (i.e. the MI saturates quickly as a function of population size). To avoid an overestimation

167 of information from high-dimensional population data (due to the limited sampling bias), we first decoded

168 the stimulus from single trial responses using a support vector machine (SVM) based decoder (Fig. 4A)

169 before computing the MI (Ince et al., 2010b, 2010a; Quian Quiroga and Panzeri, 2009). To verify that this

170 method did not introduce a positive bias, we computed the information in response to an artificial

171 uninformative stimulus set (same PSTH for all stimuli, independent Poisson spiking), which yielded near-

172 zero MI values (Fig. 4C). The performance of the SVM provided significantly better results (correctly

173 predicting $94 \%$ of the stimuli), than linear $(79 \%)$ or quadratic $(80 \%)$ decoders. However, better decoders 
174 than the SVM may still exist, and the information calculated here therefore constitutes a lower bound on

175 the available information in the population data. Also, it should be noted that the present timing code does

176 not automatically include the rate code, since only the first spike is considered (see Materials and Methods).

\section{Contribution of timing/rate reliability for information recovery}

178 While a well-defined stimulus can be provided in vitro, the details of the L4 population activity cannot be

179 controlled. From the perspective of information transmission in single trials, the most important property

180 of the neural response is the reliability across trials. We utilized the barrel column in silico (Huang et al.,

181 2020) to investigate the influence of the encoding strategy in L4 (reliability of spike timing and spike count)

182 on the information transfer to L2/3.

183 We first considered three extreme cases of encoding in L4, with a more systematic exploration in 184 the following section. In the first case ('Rate + Poisson'), stimuli are encoded only by the population PSTH,

185 but spikes across trials and neurons are drawn with Poisson statistics. In the second case ('Rate + Trial

186 Reliability'), stimuli are encoded by the population PSTH in L4, but also by the spike timing and count of

187 its individual neurons (i.e. the spike trains were identical for each trial with the same stimulus conditions).

188 Within the constraints of the experimentally observed PSTHs, these two cases constitute the lower and

189 upper bound of trial-to-trial reliability in L4. In the third case ('No Rate + Trial Reliability'), the population

190 PSTH carries no information about the stimulus, but all information about the stimulus is encoded in the

191 spike timing and count of individual neurons. The latter case is added for comparison with the other two

192 encoding paradigms. Here, the population PSTH does not distinguish between stimuli, which happens for

193 instance for texture recognition tasks (Arabzadeh et al., 2005). These three cases are illustrated in the insets

194 of Fig. 4D-F. More details regarding the construction of these cases are given in Materials and Methods.

195 In the 'Rate + Poisson' case, information transfer is overall low, with interneurons providing a

196 superior readout of the information about the stimulus in L2/3 compared to excitatory neurons, both when 
197 the information was decoded in rate and in timing (Fig 4D right, light and solid colors respectively, 198 excitatory (red) vs. inhibitory (blue) neurons). While timing and rate codes are similarly efficient in

199 interneurons, substantially larger populations of excitatory neurons are required to decode information in 200 rate than in time. Given that the timing of the stimulus is only present on the population level in L4, the 201 dominance of this temporal readout in L2/3 is remarkable. Assuming the emulated stimuli in silico 202 approximate the stimuli in vitro with high accuracy (see Figs. 1 and 2), the 'Rate + Poisson' coding does 203 not reflect the L4 encoding scheme in the present experiments.

204 In the 'Rate + Trial Reliability' case, the information transfer is overall substantially higher than in 205 the 'Rate+Poisson' case (Fig. 4E right). In this condition the number of neurons required to recover the full 206 stimulus information is the lowest $(\sim 25)$ of the three cases. This was expected, since in this case two sources 207 of information - rate and timing - are used in the encoding of the stimulus. Remarkably, both cell-types and 208 both decoding strategies yield very similar information values here, suggesting this encoding strategy is 209 optimal for information transfer.

210 In the 'No Rate + Trial Reliability' case, the information transfer is intermediate between the two 211 preceding cases. Here, the stimulus is only encoded by the responses of individual L4 neurons, not by the 212 population PSTH. Interestingly, the opposite from the 'Rate + Poisson'-case can be observed here in the 213 decoding efficiency between L2/3 cell-types: contrary to the 'Rate + Poisson'-case, where interneurons 214 transfer more stimulus information, here excitatory neurons become substantially more efficient at 215 representing information (compare Fig. 4D to 4F).

216 In summary, the availability of stimulus information in L2/3 spike trains is highly dependent on 1) 217 the encoding properties of L4, 2) the decoding strategy in L2/3 and 3) the identity of the L2/3 neuronal 218 populations (inhibitory or excitatory, for a summary see Table 1). Information about the stimulus in the 219 spikes of L4 single units is best recovered by L2/3 excitatory neurons (Fig. 4F), given that there is a 
220 reasonable trial-to-trial reliability. Next, we systematically modulated the information content in L4 spike

221 trains to investigate the consequences for L2/3 information availability.

\section{Population and single unit information selectively influence inhibitory or excitatory cells}

223 While we only considered the extreme cases of L4 encoding above, realistic encoding will necessarily cover

224 a range of cases between these extremes. Different stimuli will often, but not always, lead to different

225 population PSTHs (but different surface textures may well lead to similar population PSTHs while finely

226 modulating single unit responses (Arabzadeh et al., 2005). Conversely, even in cases where the population

227 PSTH carries substantial information about the stimulus identity, spiking may well not be Poisson, but more

228 reliable (especially at the response onset see e.g. (Amarasingham et al., 2006)).

229 We investigated the contributions from the population and the single unit separately. Information

230 on the population level was represented as the average PSTH of the population (see Figure 6A1). Stimulus

231 information was encoded in L4 spike trains either as timing or rate differences. Timing differences were

232 implemented as shifts of the PSTHs $\left(\Delta_{\mathrm{T}}\right)$, whereas firing rate differences were implemented as rate factors

233 between the PSTHs $\left(\Delta_{C}\right)$. If $\Delta_{T}=0 \mathrm{~ms}$ and $\Delta_{C}=1$, then no information is contained in the population PSTH.

234 Conversely, if $\Delta_{\mathrm{T}}=4 \mathrm{~ms}$ and $\Delta_{\mathrm{C}}=4$, the combined differences between the PSTHs are similar to the

235 experimentally observed ones. These parameters allow us to study the susceptibility of L2/3 neurons to the

236 different encoding strategies of L4 neurons (examples of spike patterns are shown in Fig. 5A1 above the

237 PSTHs, 10 trials each).

238 For decoding using spike times, inhibitory neurons exhibited a substantially greater susceptibility

239 to variations in the distinguishability of stimuli on the L4 population level compared to excitatory neurons

240 (blue vs. red, Fig. 5A2). This was true for both variations in time $\left(\Delta_{T}\right)$ and rate $\left(\Delta_{C}\right)$ in L4. Similarly, for

241 rate decoding, inhibitory neurons were more susceptible to changes in rate than excitatory neurons (Fig. 
242 5A3). Timing had little effect on rate decoding, since this corresponds mostly to a shift in the analysis

243 window (unrestricted here), with no change in rate information in L2/3.

244 Next, we considered the influence of various levels of single unit information on the information

245 availability in L2/3. Here, the population PSTH is kept fixed, but the temporal and count reliability are

246 varied on a neuron-to-neuron basis (see Fig. 5B1). The timing reliability was varied by introducing a

247 temporal jitter to individual spikes across trials $\left(\mathrm{SD}_{\mathrm{T}}\right)$, while contracting spiking patterns to remain

248 consistent with the population PSTH. Count reliability was varied selectively by a linear transition between

249 a completely reliable and a Poisson model, while maintaining the population PSTHs. This was done by

250 shifting spikes between neurons while maintaining the temporal variability across neurons.

$251 \quad$ For decoding using spike times, excitatory neurons showed much greater susceptibility to single

252 unit differences in reliability in L4, both for rate and time (Fig. 5B2). Interestingly, this carried over to the

253 rate decoding to an even greater degree, which may be the domain of action for inhibitory neurons (Fig.

254 5B3).

255 In summary, L2/3 excitatory neurons are much more sensitive than L2/3 inhibitory neurons to the 256 spike timing of L4 single neurons, whereas the information encoded by the population PSTH in L4 is carried

257 mostly by inhibitory L2/3 neurons. Hence, we propose that the inhibitory and excitatory populations

258 perform stimulus decoding in parallel, extracting stimulus information from distinct features in L4 activity

259 (see Table 1). Together they have the ability to represent the entire information efficiently in small

260 populations.

\section{Information recovery occurs rapidly within a few milliseconds}

262 Information processing in the sensory cortices is under severe temporal constraints, especially in S1, where

263 the sensory input is tightly integrated with the motor output for the purpose of precise and adaptive whisking

264 control (Li et al., 2015; Proville et al., 2014; Voigts et al., 2015, 2008). The state of processing at a given 
265 time can be estimated by computing the mutual information over limited time windows, which 266 progressively include a larger proportion of the neural response (Fig. 6A, all excitatory and inhibitory 267 neurons separated for a single trial). In combination with varying the group size, we can thus obtain a 268 'neurotemporal' overview over the process of information availability in L2/3 as a function of neuronal 269 class.

We consider three different encoding strategies by L4, 'Rate + Poisson', 'Rate + Trial Reliability' 271 and 'No Rate + Trial Reliability' as in the previous section (Fig. 6B-D). In the 'Rate + Poisson' case, the 272 mutual information begins to increase with interneurons leading over excitatory neurons (Fig. 6B, left, 273 group size $=10$ cells) around $12-14 \mathrm{~ms}$ after stimulus onset (in L4). The inhibitory neurons reach maximal 274 stimulus information, and do not achieve full stimulus information. Groups of inhibitory neurons encode 275 more information than excitatory neurons, independent of the time relative to the stimulus onset and almost 276 independent of group size (Fig. 6B, right).

277 For the 'Rate + Trial Reliability' encoding condition in L4, the difference in the information content 278 between cell types in L2/3 is small, with inhibitory neurons carrying slightly more information at early peri279 stimulus times and across all group-sizes (Fig. 6C, right). The difference in onset timing renders the 280 information content of the inhibitory neurons higher only during the initial 1-2ms after response onset, due 281 to the earlier response times of the inhibitory neurons (Fig. 6C, left).

282 In the 'No Rate + Trial Reliability' condition, the times when the information content increases are 283 very comparable for excitatory and inhibitory neurons. However, after a few milliseconds, excitatory 284 neurons prevail over inhibitory neurons. This advantage is preserved over time, whereas the difference in 285 information content as a function of group size is strongly reduced, with inhibitory neurons eventually 286 catching up with excitatory neurons (Fig. 6D, right).

287 In summary, the representation of stimulus information in L2/3 is rapidly completed within only a 288 few milliseconds (3-5) after response onset. Which neurons, i.e. excitatory or inhibitory, carry more 
289 stimulus information is determined by the encoding strategy in L4 (corresponding potentially to different

290 types of stimuli), but not much on the peri-stimulus time. As before, pure rate coding on the level of L4 is

291 identified as an insufficient coding strategy, as it does not fit our experimental results of almost complete

292 information recovery.

\section{Discussion}

294 We demonstrated that although the intracellular information transfer, i.e. the PSP-to-action potential 295 transformation, results in a significant loss of information about the stimulus, local networks can overcome 296 this loss by integrating information from a small, experimentally tractable, number of neurons. Therefore, 297 the somatic PSPs received by a single cortical neuron contain nearly complete information about the 298 stimulus, even several synapses away from the sensory periphery. The efficiency of such information 299 recovery is determined by a conjunction between the encoding scheme, neuronal class and decoding 300 strategy. Excitatory and inhibitory cells take complementary roles in carrying information in single unit or 301 population activity, respectively.

\section{Contribution of temporal coding in somatosensory cortex}

Encoding information on short temporal scales can enrich the information content of neural activity

304 relative to coarser average rates (Bialek et al., 1991; Bialek and Rieke, 1992). There has been a long

305 discussion about whether the brain uses such a 'spike code' or 'rate code' (for a review, see Brette, 2015).

306 It has been argued that since cortical networks are both noisy and very sensitive to perturbations, a rate

307 code is the only way to perform reliable computations (London et al., 2010, but see Denève and Machens,

308 2016). However, others have pointed to the presence of temporally encoded information in the

309 somatosensory (Alenda et al., 2010; Panzeri and Diamond, 2010; Petersen et al., 2001) and other sensory

310 cortices (Kayser et al., 2012, 2010). In particular, the timing of the first (few) spike(s) in response to a 
311 stimulus conveys much of the information present in a spike train (Gollisch and Meister, 2008; Johansson

312 and Birznieks, 2004). Consistently, we find that the majority of the information in the PSP is encoded in its

313 timing. However, the timing of a spike in response to such a PSP is substantially more variable than the

314 PSP timing, such that only a small proportion of the information in the PSP is transferred to the spike

315 (Fig. 2). The amount of information loss could even be more substantial in vivo in the presence of

316 background ongoing activity. On the population level, we find again that the temporal information is highly

317 relevant during information recovery. In agreement with the previously observed importance of the first

318 spikes, we find that the information content in the population asymptotes within $5 \mathrm{~ms}$ after the first spike

319 in local populations, consistent with the time-scales of neuronal read-out in whisker cortex estimated before

320 (5-8 ms, (Stüttgen and Schwarz, 2010)).

321 The temporal information described above can be fully characterized by single neuron variations

322 in rate, and hence does not include higher order temporal codes, such as the pattern of inter-spike intervals.

323 Due to the sparse response nature of supragranular excitatory neurons, such a fine-grained higher order

324 temporal code could only exist in the inter-spike intervals of inhibitory neurons or spike-patterns across

325 multiple (excitatory or inhibitory) neurons. The term temporal code is however still appropriate for our

326 results, since the time scales of the response are not only reflecting dynamics in the stimulus, but correspond

327 to intrinsic computations of the neural network (Nemenman et al., 2004).

328 For the present dataset, Shannon's mutual information was computed with responses aligned to the

329 stimulus onset. Recent work by Panzeri and colleagues (Panzeri et al., 2010; Panzeri and Diamond, 2010)

330 have pointed out that such a reference time is not necessarily available to a decoder in S1. How would a

331 change to an internal reference time, such as the efference copy of the whisking signal (Crochet et al., 2011;

332 Crochet and Petersen, 2006; Poulet and Petersen, 2008) or a population-based timing (e.g. the "Columnar

333 Synchronous Response", CSR, event defined by (Panzeri and Diamond, 2010)) affect the present results?

334 Assuming that the population response can be approximated by a set of individually recorded neurons (as 
335 in (Panzeri et al., 2010; Panzeri and Diamond, 2010)), the influence of such an intrinsic reference on our

336 results would be only minor, since the relative timing - and thus the relative trial-to-trial variability in timing

337 - would be the same as in the stimulus locked case. Hence, the information content would not be modified.

338 If, on the other hand, synchronization between neural groups occurs, results could be significantly

339 influenced, since then variability could be transferred from spikes to PSPs (in which case the alignment

340 would be based on the near-synchronous CSR). According to Petersen and colleagues (Petersen et al.,

341 2001), covariability, measured as noise correlation, was assessed to be $\sim 0.1$, and subsequent studies have

342 found even lower values (Renart et al., 2010), suggesting that stimulus-independent synchronization is not

343 substantial (note however the results of (Franke et al., 2016)

\section{High information availability and multiplexed codes}

345 To understand 'how the brain works', we need to understand what the neural computations are that

346 make an animal interact with its environment, i.e. how neural activity is transformed from the low-level

347 response, to perceptual input, to the high-level neural activity that generates behavior (Eliasmith and

348 Anderson, 2002). For instance, perceptual invariance (an object can be recognized as one and the same

349 under different circumstances) and selectivity (an object can be distinguished from other, similar objects)

350 need to be explained by any consistent theory of perception (Seung and Yuste, 2012). A model of how

351 increasingly abstract features can be recognized by neural networks along the sensory axis was already

352 explained by for instance the perceptron-model (Rosenblatt, 1958; Seung and Yuste, 2012). When neurons

353 in each processing layer respond to only a single, increasingly abstract, preferred feature, they disregard

354 necessarily a lot of information. Therefore, on the single-neuron level, the transformation from input to

355 output is expected to be very sparse, and 'lossy'. However, whether on a population level it is necessary to

356 be able to fully reconstruct the stimulus, remains an open question. We have shown here that the entire

357 stimulus information is maintained in layer $2 / 3$ of the barrel cortex and encoded by local populations in a 
distributed fashion. This information can be recovered already on the basis of a small subset of neurons ( 10-20, if single unit information is present) on short time-scales ( $\sim 5 \mathrm{~ms}$ relative to response onset), ensuring a lossless representation of the sensory world in real-time, i.e. before the next sensory information

361 arrives from the periphery (in the case of active tactile exploration in freely behaving rodents, the inter362 contact intervals are $>30 \mathrm{~ms}$ (Voigts et al., 2015, 2008)). In different setups (Dalgleish et al., 2020), in 363 mouse visual cortex (Sriram et al., 2020) and in salamander retina (Marre et al., 2015), comparable values 364 have been reported. This suggests that the full stimulus information is needed for the computations at 365 several levels. Combined with the single neuron selectivity, our results suggest that this network performs 366 a form of coordinate transformation (Denève and Pouget, 2003). However, what the exact nature of the 367 computations of this and downstream networks is, remains an open question.

The neural activity of excitatory neurons in cortical layers $2 / 3$ is generally considered to be sparser than in Layer 4 (see (Barth and Poulet, 2012) for a review, although the evidence is not yet fully conclusive).

370 This sparsity has been linked to higher selectivity of encoding, in terms of fewer, more specific features 371 represented per neuron. This increased selectivity could be the reason for the observed information loss 372 during the transformation of PSPs to spikes. It has been argued that the sparsity of the transformation of 373 presynaptic spike trains to PSPs to postsynaptic spikes is the result of optimal non-linear processing: only 374 redundant information, that has been gained before and can be predicted from previous activity, is 375 discarded, and postsynaptic neurons only respond to 'new' information (Denève, 2008; Ujfalussy et al., 376 2015). Our result that the postsynaptic membrane potential still contains the full stimulus information, is in 377 agreement with this argument, and the observation that most information is contained in the first spikes 378 mentioned before could also be explained this way. However, whether the information that is lost in the 379 spike-generating process is truly 'discarded' information, or, contrarily, redundant information, depends on 380 the presumed decoding of the neuron: which information is redundant or essential depends on the message 381 that needs to be conveyed. 
The distributed persistence of complete stimulus information could provide a practical solution to

383 one of the classical dilemmas of neural encoding: the compatibility between a specific feature and the

384 context of the entire stimulus space. Concretely, a readout neuron in L2/3 may have privileged access to L4

385 neurons selective for one type of feature, with in addition access to a wide range of inputs from a random

386 subset of the population. It could thus act as a comparator and evaluate the dominant feature in relation to

387 a representation of the entire stimulus. This becomes especially relevant in the case of multiple concurrent

388 stimulations on different whiskers, corresponding to the natural situation an animal is exposed to during

389 active exploration (Voigts et al., 2015). In this case, multiple signals (i.e. the signals from multiple whiskers,

390 that carry different spatial and temporal information) have to be processed by a single population of

391 neurons. If this population can be separated into independent-subpopulations, this implies that a population

392 consists of multiple channels (in the information-theory sense), but if this is not the case, multiple signals

393 are coded by a single population, so the code becomes multiplexed: a single channel (population) carries

394 complementary information through different codes. The observation that multiple subsets of neurons carry

395 complete stimulus information and the observation that spike timing and firing rate of the same population

396 (channel) can contain independent information about the stimulus hints at such multiplexing ((Panzeri et

397 al., 2001; Quian Quiroga and Panzeri, 2009), for a review, see (Panzeri et al., 2010)). Our finding that

398 different postsynaptic populations can decode the timing-encoded and rate-encoded information shows that

399 the information from both coding schemes (rate and timing by inhibitory and excitatory neurons) of such

400 multiplexed encoded information can also be used by the brain for further processing in later stages.

401 Information in inhibitory populations can then be forwarded by for instance disynaptic (dis)inhibition and

402 the modulation of firing rates or spike probabilities of excitatory populations. Multiplexed codes have been

403 discussed recently in the context of local field oscillations (Alenda et al., 2010), and the presence of

404 selective and general information as described herein may provide an additional example of multiplexing

405 (Fig. 7). 
Given the information content across the excitatory and inhibitory neural populations calculated

407 herein, we speculate that distinct tactile features are encoded by rate and timing of spiking during

408 information encoding and decoded by excitatory and inhibitory neurons separately (Fig. 7A). If an animal

409 were to use its whiskers to locate a tactile target in space for example (Celikel and Sakmann, 2007; Peron

410 et al., 2015), this model predicts that inhibitory neurons would carry the largest amount of information

411 during the first contact as the animal detects the edge of the tactile target. Similarly, at the detection of a

412 contact during passive whisking (Clem et al., 2008), inhibitory neurons would preferentially respond to the

413 onset of touch, serving as an edge detector. The information content of different signals within the L4-to-

414 L2/3 channel is temporally constrained as the animal continues to explore its immediate environment, and

415 makes additional whisker contacts with the tactile target (Fig. 7B; (Voigts et al., 2015, 2008)), presumably

416 to predict object distance and extract additional surface feature information about the target. With the

417 change of whisking pattern, the sensory history and the statistics of the local network activity, relative

418 information in the excitatory population will eventually dominate the neural representation of touch (Fig.

419 7C).

How to convey stimulus information both lossless and efficiently, and how this depends on physical

421 properties such as network connectivity and node (neuron) properties, is an important open question in

422 network science, and neuroscience specifically (Maheswaranathan et al., 2018; Mastrogiuseppe and

423 Ostojic, 2017), in which computational models like the one we present here (Huang et al., 2020) play an

424 invaluable role. Recently, it has been shown that there is a trade-off between the sparsity and the amount

425 of recovered information in neural coding (Billings et al., 2014): lossless coding is only possible if the

426 connectivity in the network is not too sparse. Specifically, the authors showed that optimal connectivity

427 included only a few excitatory synapses and strong inhibition. Moreover, activity-dependent thresholds 
428 appear to play an invaluable role in such efficient information transmission (Billings et al., 2014; Huang et

429 al., 2016).

For future in vivo studies, an important question will be, whether complete information

431 representation persists if larger stimulus sets/spaces are considered, since it is expected that the

432 dimensionality of the response, and hence the number of neurons needed for complete information

433 recovery, depends on the complexity of the stimulus (Gao and Ganguli, 2015). Due to the requirements of

434 accurate estimation of mutual information, we had to restrict the stimulus space to four stimuli in the context

435 of whole-cell recordings (leading to $\sim 300$ trials per recorded cell). Note, however, that even under these

436 conditions, trial-to-trial variability could have prevailed and prevented complete stimulus reconstruction on

437 the single neuron and population level. Moreover, the present results can only provide a lower bound on

438 the available information, since not all possible codes were explored and the decoding step between

439 stimulus and response renders all results lower bounds (Quian Quiroga and Panzeri, 2009). In contrast to a

440 previous study in the auditory cortex (Ince et al., 2013), we find that more complex decoding methods

441 provide an improved decoding quality and hence more mutual information. Concretely, support vector

442 machine decoding with radial basis functions provided superior performance (94\%) than either diagonal

443 linear (77\%), linear (79\%), or quadratic (80\%) decoders. In order for the neural system to achieve this

444 quality of decoding, it would, however, need to have readout mechanisms which use decoding strategies

445 beyond linear or quadratic combinations.

\section{Predictions for cell-type specific coding strategies}

447 The cortical population of neurons is composed of various cell-types, which differ in their morphology,

448 location and physiology (De Kock et al., 2007; Narayanan et al., 2015; Oberlaender et al., 2012; Staiger et

449 al., 2015). These differences suggest distinct roles in information processing, some of which have recently 
450 been elegantly elucidated (Ko et al., 2011). Coarsely, on the level of their firing patterns, inhibitory neurons

451 can be distinguished from excitatory neurons, by more dense responses, based on a greater convergence of

452 connections (reviewed in Harris and Mrsic-Flogel (2013)). The connectivity in the present model was set

453 in precise accordance with the latest results from the literature from identified, pairwise recordings (see

454 Huang et al. (2020) for detailed references), and consequently recreates these differences in firing behavior.

455 Going beyond previous work, we find the coding balance to lean to either cell class, depending on the 456 encoding strategy used in L4.

457 We explored these encoding strategies in L4, finding that excitatory neurons more effectively 458 convey information encoded in L4 single units, requiring a level of reliability in L4 beyond Poisson-spiking 459 (Figure 6B). Conversely, inhibitory neurons are more effective in carrying L4 population rate information 460 (Figure 6A). Hence, together, excitatory and inhibitory neurons make effective use of the combined 461 information in population rate and single unit responses in L4.

462 The L4 encoding is likely to depend on the stimulus condition: Many stimuli will induce time463 varying population rates, which distinguish them from other stimuli. However, exceptions exist, such as the 464 comparison of similar textures (Arabzadeh et al., 2005), which have only small differences in population 465 rate, and differ more in their fine-structure. On the other hand, temporally structured inputs (e.g. many 466 natural stimuli) lead to stronger time locking between neurons in L4 (Amarasingham et al., 2011; Litwin467 Kumar and Doiron, 2012). Based on our results, we suggest that excitatory and inhibitory neurons might 468 focus on distinct individual and population information to optimize the availability of stimulus information 469 in local networks. Since long-range projections of inhibitory neurons are rare (Thomson and Lamy, 2007), 470 the information content in the spiking of the inhibitory neurons is likely to be most relevant for local 471 processing. Testing this hypothesis will not be trivial, since the inhibitory neurons cannot be removed from 472 the network without influencing the overall network dynamics. Nonetheless, transient optogenetic 473 modulation of the rate and timing of select inhibitory neurons' activity while studying neural encoding of 
474 stimuli in the rest of the network will help to answer the question about which inhibitory neurons contribute

475 more to the transfer and recovery of information within a column (i.e. local) and across columnar networks.

In summary, the results presented here suggest that single neurons are efficient real-time encoders

479 of stimuli even several synapses away from the sensory periphery, but intracellular information transfer

480 results in a substantial loss in the information transmitted to the postsynaptic neurons. The lost information

481 can be recovered rapidly, i.e. within $20 \mathrm{~ms}$, by comparatively small numbers of neurons in local populations,

482 so that lossless information transfer along the sensory axis is ensured. The information recovery depends

483 critically on the type of the neuron as well as the coding properties of both the presynaptic and postsynaptic

484 pools of neurons, such that excitatory and inhibitory populations process complementary information about

485 the stimulus in the somatosensory cortex. 


\section{Materials and Methods}

\section{Experimental procedures}

489 Rats from either sex were used according to the Guidelines of National Institutes of Health and were

490 approved by the local Institutional Animal Care and Use Committee. All data can be found in this online 491 repository: https://doi.org/10.34973/59my-jm24, the relevant code can be found here: 492 https://github.com/DepartmentofNeurophysiology/Information-transfer-and-recovery-for-sense-of-touch-

493 code-for-figures

\section{In vitro recordings}

495 In vitro whole-cell current-clamp recordings were performed in acutely prepared slices of the barrel cortex 496 between P18-21, after maturation of evoked neurotransmitter release (Martens et al., 2015) as described 497 before (Allen et al., 2003; Celikel et al., 2004; Clem et al., 2008). Oblique thalamocortical slices (300 mm, 498 (Finnerty and Connors, 2000)) were cut $45^{\circ}$ from the midsagittal plane in chilled low-calcium, low-sodium 499 Ringer's solution (in mM; sucrose, 250; $\mathrm{KCl}, 2.5 ; \mathrm{MgSO}_{4} .7 \mathrm{H}_{2} \mathrm{O}, 4 ; \mathrm{NaH}_{2} \mathrm{PO}_{4} . \mathrm{H}_{2} \mathrm{O}, 1$; HEPES, 15; D-(+)500 glucose, $11 ; \mathrm{CaCl}_{2}, 0.1$ ). Slices were first incubated at $37^{\circ} \mathrm{C}$ for 45 minutes and were subsequently kept in 501 room temperature in carbonated $(95 \% \mathrm{O} 2 / 5 \% \mathrm{CO} 2)$ bath solution $(\mathrm{pH} 7.4$, normal Ringer's solution: in $502 \mathrm{mM}, \mathrm{NaCl}, 119 ; \mathrm{KCl}, 2.5 ; \mathrm{MgSO}_{4}, 1.3 ; \mathrm{NaH}_{2} \mathrm{PO}_{4}, 1 ; \mathrm{NaHCO}_{3}, 26.3 ; \mathrm{D}-(+)$-glucose, $\left.11 ; \mathrm{CaCl}_{2}, 2.5\right)$.

503 Visualized whole-cell recordings were performed using an Axoclamp-2B amplifier under IR-DIC 504 illumination. A custom-made tungsten bipolar extracellular stimulation electrode (inter-tip distance 150 505 micrometer) was placed in the lower half of a L4 barrel. Stimulation protocol was as described before 506 (Huang et al., 2016). In short, $10 \mathrm{~ms}$ long current pulses were delivered using a bipolar electrode located in 507 the lower half of a mystacial whisker's barrel. The pulses were square and had equal maximal amplitude 508 although the rising phase of the stimulus had different slopes. It took $0,2,4$ or $6 \mathrm{~ms}$ for the pulse to reach 
509 the maximum amplitude for stimulus (S)1, S2, S3 and S4, respectively. All intracellular recordings (pipette

510 resistance 3-4 MOhm) were performed in L2/3, orthogonal to the stimulation electrode within 150-300 $\mu \mathrm{m}$

511 of the cortical surface. The internal solution ( $\mathrm{pH} 7.25$ ) consisted of, in $\mathrm{mM}$, potassium gluconate, $116 ; \mathrm{KCl}$,

512 6; NaCl, 2; HEPES, 20 mM; EGTA, 0.5; MgATP, 4; NaGTP, 0.3. For whole cell recordings, putative

513 excitatory cells were selected based on pyramidal shaped somata, apical dendrites and distal tuft orientation,

514 and regular pattern of spiking to somatic current injections (500 ms; data not shown). Data was low-pass

515 filtered $(2 \mathrm{kHz})$, digitized at $5 \mathrm{kHz}$ using a 12-bit National Instruments data acquisition board and acquired

516 using Strathclyde Electrophysiology Suite for offline data analysis.

\section{In vivo recordings}

518 In vivo whole-cell current-clamp recordings were performed under ketamine/xylazine anesthesia at P28-

519 30. Anesthesia was induced using $100 \mathrm{mg} / \mathrm{kg}$ (ketamine) and $10 \mathrm{mg} / \mathrm{kg}$ (xylazine) mixture and maintained

520 with intraperitoneal ketamine-only injections (20\% of the initial dose) as necessary. Upon complete loss

521 of facial and hind-limb motor reflexes, the skull was exposed. A head-bolt was fixed posterior to lambda

522 using cyanoacrylate and was used to immobilize the animal during experiments.

523 The surface over the primary somatosensory cortex (from Bregma, $-0.5 \mathrm{~mm}$ to $-2.5 \mathrm{~mm}$, from Midline -

$5242.5 \mathrm{~mm}$ to $-4.5 \mathrm{~mm}$ was thinned using a dental drill. The surface was kept moist with a thin layer of low-

525 viscosity mineral oil to maintain the transparency of the thinned skull. Cortical representation of the D2

526 whisker was localized in the contralateral hemisphere using intrinsic optical imaging as described before

527 (Stewart et al., 2013) while deflecting individual whiskers using piezoelectric actuators as described

528 elsewhere (Celikel et al., 2004). The skull above the center of mass of the functional whisker representation

529 was punctured using a 28 gauge needle to allow patch electrodes to access the cortical region of interest.

530 All electrode penetrations were perpendicular to the cortical surface. In vivo whole-cell recordings were

531 performed as described before (Margrie et al., 2002) with recording electrodes (6-7 MOhm) filled with the 
532 same intracellular solution used in slice experiments. Two different whisker deflection protocols were

533 used: During optical mapping experiments single whiskers were deflected along the dorsoventral axis at 5

$534 \mathrm{~Hz}$ with $8^{\circ}$ deflections for 20 times with an inter-trial interval of $20 \mathrm{sec}$ (Stewart et al., 2013). During

535 electrophysiological recordings single dorsoventral whisker deflections were delivered at $0.2 \mathrm{~Hz}$ for 200

536 times. In each trial $4^{\circ}$ whisker deflections were delivered at $10 \mathrm{~Hz}$ for $1 \mathrm{~s}$. Throughout the experiment the

537 animal's core body temperature was maintained at $36.5 \pm 0.5^{\circ} \mathrm{C}$.

\section{$538 \quad$ Data analysis}

539 All analyses were performed off-line in Matlab (Mathworks, Inc), the code for the figures can be found

540 online: https://github.com/DepartmentofNeurophysiology/Information-transfer-and-recovery-for-sense-

541 of-touch-code-for-figures. Raw voltage traces were smoothed using running window averaging (1ms

542 window size) and the following variables were calculated for all evoked responses: Onset time (Ot, in ms):

543 Latency of the postsynaptic potential (PSP) onset in respect to onset of the stimulus; Rise time (Rt, in ms):

544 Time it takes for the membrane to reach $90 \%$ of the PSP amplitude relative to the onset of PSP; PSP slope

545 ( $\mathrm{Sl}$, in $\mathrm{mV} / \mathrm{ms}$ ) between 10-90\% of the PSP amplitude and amplitude of the EPSP (Amp, in $\mathrm{mV}$ ). If the

546 trial included an action potential, the peak of the EPSP was set to the spike threshold (Vt). The spike

547 threshold was defined as the membrane potential value at which the second derivative of the membrane

548 potential reached a maximum as described before (Wilent and Contreras, 2004). In slice recordings, resting

549 membrane potential $(\mathrm{Vm}$, in $\mathrm{mV})$ was calculated as the average membrane potential in a $40 \mathrm{~ms}$ time window

550 prior to the stimulus onset. For in vivo recordings the same time window was used but the sweep was

551 included in the data analysis only if the variance of the membrane potential was $<0.5 \mathrm{mV}$ during the time

552 window. For those sweeps in which a spike was observed, the spike threshold and spike latency (St) were

553 also calculated. 


\section{Mutual information analysis for single neurons.}

555 Only cells with more than 250 acceptable sweeps (summed across all stimulus conditions) were 556 used to perform Shannon information analysis. The mutual information (MI) between any two 557 variables $S, R$ can be calculated as

$$
I(S ; R)=H(R)-H(R \mid S),
$$

559 in which $\mathrm{H}$ is the entropy of a given variable $\mathrm{R}$ :

$$
H(R)=-\sum_{i=1}^{n} p\left(r_{i}\right) \log _{2}\left(p\left(r_{i}\right)\right)
$$

and $\mathrm{H}(\mathrm{R} \mid \mathrm{S})$ is defined as

$$
H(R \mid S)=-\sum_{i=1}^{n} p\left(s_{i}\right) \sum_{r} P(r \mid s) \log _{2} p(r \mid s)
$$

where i ranges over the stimulus/response types. Note that the stimulus entropy shows a small variability due to rejected trials. Similarly, the mutual information between one variable $\mathbf{S}$ and multiple $\mathbf{R}$ (joint mutual information) can also be calculated using equation (1). In this case, the synergistic effect of $\mathbf{R}$ can be expressed as the difference between the linear sum of the mutual information between $\mathrm{S}$ and each individual

$567 \mathrm{R}$ and the joint information $\mathrm{I}(\mathrm{S} ; \mathbf{R})$ :

$$
\operatorname{Syn}(S ; \mathbf{R})=I(S ; \mathbf{R})-\sum_{i=1}^{n} I\left(S ; R_{i}\right)
$$

Information calculations were performed using the Information Breakdown toolbox (Magri et al., 2009) in Matlab (Mathworks. Inc). In short, each variable was first digitized using the equal space ('eqspace') binning method with 7 bins. The effect of different binning methods as well as the number of bins on MI values are

572 also explored (Fig. S1). In the analysis based on the 'eqpop' binning method, the size of individual bins 573 was modified so that a roughly equal number of observations was placed in each bin, instead of keeping

574 the size of individual bins constant. Because in most trials only one spike was observed, only the first spike 575 was considered when calculating the information in St. Thus, the spike latency St can be digitized to a single word, which has (number of bin +1 ) possible outcomes, instead of a binary list which could have 
$5772^{\wedge}$ (number of bin) possible values. Shuffle correction combined with Panzeri-Treves (Panzeri and Treves,

578 1996) bias correction was used to perform all information calculations for neural recordings (note that

579 shuffle corrections can introduce a small source of variability, which can be seen for instance in comparing $580 \mathrm{I}(\mathrm{S}, \mathrm{PSP})$ in Figure 2A with and H(S) or with the joint MI in 2C, or that can lead to an error bar above the 581 stimulus entropy or below 0). The performance of the algorithm was evaluated by randomly selecting a 582 subset of trials to calculate the mutual information (I(S;PSP), I(PSP;Vt) and I(PSP;St)), and subsequently 583 checking the number of trials (Ns) needed for the calculated information values to reach asymptote (Fig. 584 S2). When the 'eqspace' binning method was used, all information values reached asymptote after Ns > 70, 585 well below the average Ns in the present data set (124 \pm 33.2 (range: $78-220)$ stimulus repetitions per 586 stimulus).

\section{Calculation of minimum observation size:}

588 An essential step in the information calculation method listed above is the estimation of the stimulus589 response probability distributions from the experimental data. Following Panzeri and colleagues (Ince et 590 al., 2010b) we calculated the number of experimental trials per stimulus condition, Ns, to be $\sim 32$ times 591 larger than the number of possible response pattern, $R$, to get an accurate estimation $(\mathrm{Ns} / \mathrm{R} \approx 32)$. This also 592 means that to accurately estimate information between the subthreshold responses (Am, Sl, Ot, all binned 593 to 7 bins) and the stimulus, $32 \times 7 \times 7 \times 7=10976$ trials/ stimulus $=91 \mathrm{~h}$ continuous recordings will be needed.

594 Given technical infeasibility of maintaining whole cell access for the designated period we performed bias 595 corrections to account for the upward bias in information estimation with limited sample sizes (see (Ince et 596 al., 2010b) and (Victor, 2009) for further discussion). Methods like quadratic extrapolation (QE), Panzeri597 Treves (PT) correction (Panzeri and Treves, 1996) and Nemenman-Shafee-Bialek (NSB) t experiments and $59894 \pm 25.6$ (range, 60-146) trials/stimulus for the in vivo whole-cell recordings. 


\section{Mutual information analysis for multiple neurons}

600 For multi-neuron MI analysis we followed the approach to first decode and then estimate the MI between

601 stimuli and the confusion matrix of the decode (Ince et al., 2010b; Panzeri and Diamond, 2010; Quian

602 Quiroga and Panzeri, 2009) using support vector machine (SVM) in MATLAB with radial basis functions

603 as the kernel transform. We utilized 90/10\% cross validation during decoding to obtain an estimate of the

604 generalized performance of the decoder. SVM decoding outperformed other decoders with an average

605 performance of 94\%, compared with some other decoders (diagonal linear (77\%), linear (79\%), quadratic

$606(80 \%)$ ). The use of an intermediate decoder ensured that the calculation was bias free (given that we observe

607 the correct value of 0 bits for an uninformative set of stimuli, with otherwise very similar properties (see

608 Fig. 3G)), but came at the expense of lower bound in MI estimates since a (potentially existing) better

609 decoder would improve the MI.

610 For the in-vitro recordings we first had to generate bootstrapped populations of sufficient size to perform

611 the population MI calculations. In order to preserve the within-cell variability of responses across stimulus

612 and trials, we only drew bootstrap samples from the trials of each cell independently. As in the simulations

613 we drew 100 samples of groups of each population size. Curves in Fig. 4 display averages over these

614 samples.

\section{Network Simulations}

616 The reconstruction of information in the neural network was performed in an in silico model of the barrel 617 cortex. 


\section{Neural network}

619 The model included a realistic account of the number of (Izhikevich, 2004, 2003) neurons and connectivity

620 (Supplemental Table 1) within a barrel column for Layers 2/3, with inputs arriving from the L4, mimicking

621 the conditions in the in vitro/in vivo experiments. For more details on the network model, see (Huang et al., 622 2020).

623 Synaptic currents in this network were modeled by a double-exponential function. Parameters of those 624 functions (peak amplitude, rise time, half width, and pair-pulse ratio) were adjusted to match experimentally 625 measured PSPs in barrel cortex (Supplemental Table 1; see Thomson and Lamy (2007) for a review). The 626 onset latency was calculated from the distance between cell pairs; the conduction velocity of action potential 627 was set to $190 \mu \mathrm{m} / \mathrm{ms}$.

628 Differences in activation state of cortex were included in the model by setting the common initial voltage 629 and the equilibrium potential $v \boldsymbol{r}$ of all cells to $-80,-70$, or $-60 \mathrm{mV}$ in a third of the trials, thus accounting for 630 potential up- and down-states as well as an intermediate state.

\section{Synaptic input from layer 4}

632 Layer 4 stimulation was provided in the model based on population PSTHs collected extracellularly in 633 anesthetized animals in vivo (Celikel et al., 2004). We used PSTHs of principal and 1st order surround 634 whisker stimulation, as well as two linear interpolations between the two, yielding 4 stimuli with 2 bits total 635 entropy, matching the numbers in the in vitro experiments. The PSTHs only specified the population firing 636 rate in L4. We further explored population coding properties, by modifying the variability of spike timing 637 across trials. If response times and spike counts were conserved across multiple trials, spike timing and 638 counts within and between neurons start to carry additional information. 
In the 'Rate + Poisson' condition, we assumed no trial-to-trial reliability beyond that given by the

640 PSTH. Spike times were drawn based on Poisson statistics for each time with the PSTH modulating the

641 firing rate (see Fig. 4D left). This condition forms a lower bound on the transferred information between

642 L4 and L2/3, under the experimental constraints on the model. On the other extreme, in the 'Rate + Trial

643 Reliability' condition, the PSTHs varied as before, but in addition neurons emitted the same sequence of

644 spikes for every trial, preserving timing and count perfectly. This condition forms an upper bound on the

645 information transfer, since within the experimental constraints no additional variability is introduced, which

646 would reduce the mutual information. Finally, we consider the 'No Rate + Trial Reliability' condition,

647 where the population PSTHs are uninformative across stimuli, and stimulus information is only contained

648 in the spike trains of individual neurons. This case is a reference for other stimulus scenarios, where the

649 PSTH may not vary much (e.g. texture-type stimuli), and individual timing becomes more important.

650 We also explored conditions between these extremes (Fig. 5), where the information in population

651 or single neuron response was systematically varied. For the case of the population response we varied the

652 different in time and firing rate of the PSTHs for different stimuli (Fig. 5A). Time differences were

653 implemented by simply shifting the entire PSTH in time (tested shifts: $[0,1,2,3] \mathrm{ms}$ per stimulus, i.e.

654 maximum shift was $9 \mathrm{~ms}$ ). Rate differences were implemented as the fraction between the maximal and the

655 minimal stimulus (tested fractions were [1,2,3,4], where e.g. 4 corresponds to the weakest stimulus being

$65625 \%$ of the strongest stimulus at the peak of the PSTH). The case of time shift $0 \mathrm{~ms}$ and rate fraction 1 is

657 uninformative on the level of population rate. Single neuron reliability in this case was chosen as a medium

658 level of single unit reliability $\left(\mathrm{SD}_{\mathrm{T}}=3 \mathrm{~ms}, \mathrm{SD}_{\mathrm{C}}=20 \%\right)$. Single neuron reliability in response was also

659 explored in timing and rate (Fig. 5B). Starting from perfect timing and rate, we degraded the information

660 extractable from single neurons, by introducing timing variability (spike times were shifted by Gaussian-

661 distributed noise with standard deviation $\mathrm{SD}_{\mathrm{T}}$ ) and rate/count variability (spikes were deleted or added, by 
662 linearly mixing between Poisson and perfectly reliable spiking, with mixing parameter $\mathrm{SD}_{\mathrm{C}}$, denoted as \%

663 in the figure). For both procedures, the modifications were performed while keeping the population PSTH

664 approximately unchanged, i.e. for timing the overall timing distribution was contracted to keep the original

665 PSTH, and for rate, spikes were shifted between neurons, rather than only removed from individual neurons.

666 These independent variations of population and single unit responses allowed us to separate the

667 contribution of these two information sources to the information available in groups of L2/3 excitatory and

668 inhibitory neurons (see Results). 


\section{Abbreviations}

$670 \quad$ AP : Action potential / spike

671 PW : Principal whisker

$672 \quad \mathrm{H} \quad$ : Entropy

$673 \quad$ S : Stimulus

$674 \Delta_{\mathrm{T}} \quad$ : Variation in spike timing

675 L : (Cortical) layer
PSP : Postsynaptic potential

SW : Surround whisker

I : (Mutual) Information

PSTH : Peristimulus time histogram

$\Delta_{\mathrm{C}} \quad$ : Variation in spike rate

CSR : Columnar Synchronous Response

676 


\section{Acknowledgements}

678 We are grateful to Drs. Rasmus Petersen and Alessandro Treves for their comments on a previous

679 version of this manuscript, and the members of the Department of Neurophysiology for critical

680 discussions. This work was supported by the Whitehall Foundation, Alfred P. Sloan Foundation,

681 European Commission (Horizon2020, nr. 660328) and Netherlands Organisation for Scientific

682 Research (NWO-ALW Open Competition; nr. 824.14.022 and NWO Veni Research Grant, nr.

683863.150 .25 to FZ) and a Christine Mohrmann Grant to FZ. The funders had no role in study

684 design, data collection and analysis, decision to publish, or preparation of the manuscript. 


\section{Tables}

686 Table 1: Summary of information recovery results depending on L4 encoding and L2/3 decoding schemes.

\begin{tabular}{|c|c|c|c|c|}
\hline L4 encoding $\rightarrow$ & \multicolumn{2}{|c|}{ population level } & \multicolumn{2}{c|}{ single neuron level } \\
\hline L2/3 decoding $\downarrow$ & $\begin{array}{c}\text { timing } \\
\left(\Delta_{\mathrm{T}}>\mathbf{0}, \Delta_{\mathrm{C}}=\mathbf{1}\right)\end{array}$ & $\begin{array}{c}\text { rate } \\
\left(\boldsymbol{\Delta}_{\mathrm{T}}=\mathbf{0}, \Delta_{\mathrm{C}}>\mathbf{1}\right)\end{array}$ & $\begin{array}{c}\text { timing reliability } \\
\left(\mathbf{S D}_{\mathrm{T}}>\mathbf{0}, \text { shift=0) }\right.\end{array}$ & $\begin{array}{c}\text { count reliability } \\
\left(\mathbf{S D}_{\mathrm{T}}=\mathbf{0}, \text { shift }>\mathbf{0}\right)\end{array}$ \\
\hline spike times & $\begin{array}{c}\text { inhibitory neurons } \\
\text { more susceptible }\end{array}$ & $\begin{array}{c}\text { inhibitory neurons } \\
\text { more susceptible }\end{array}$ & $\begin{array}{c}\text { excitatory neurons } \\
\text { more susceptible }\end{array}$ & $\begin{array}{l}\text { excitatory neurons } \\
\text { more susceptible }\end{array}$ \\
\hline rate & no effect & inhibitory neurons & excitatory neurons \\
more susceptible & more susceptible & excitatory neurons \\
more susceptible
\end{tabular}


bioRxiv preprint doi: https://doi.org/10.1101/2020.12.08.415729; this version posted December 15, 2020. The copyright holder for this preprint

\section{Figures}

A

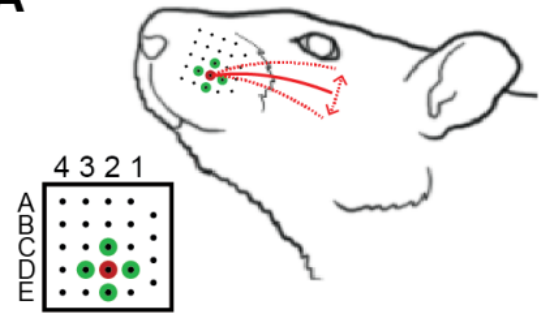

$\mathbf{E}$
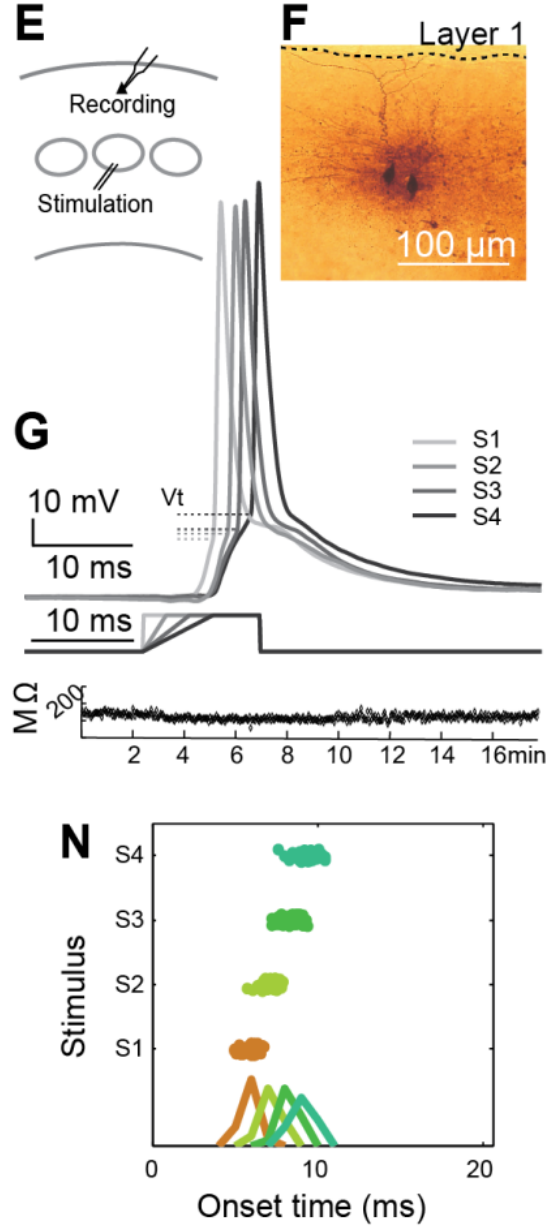
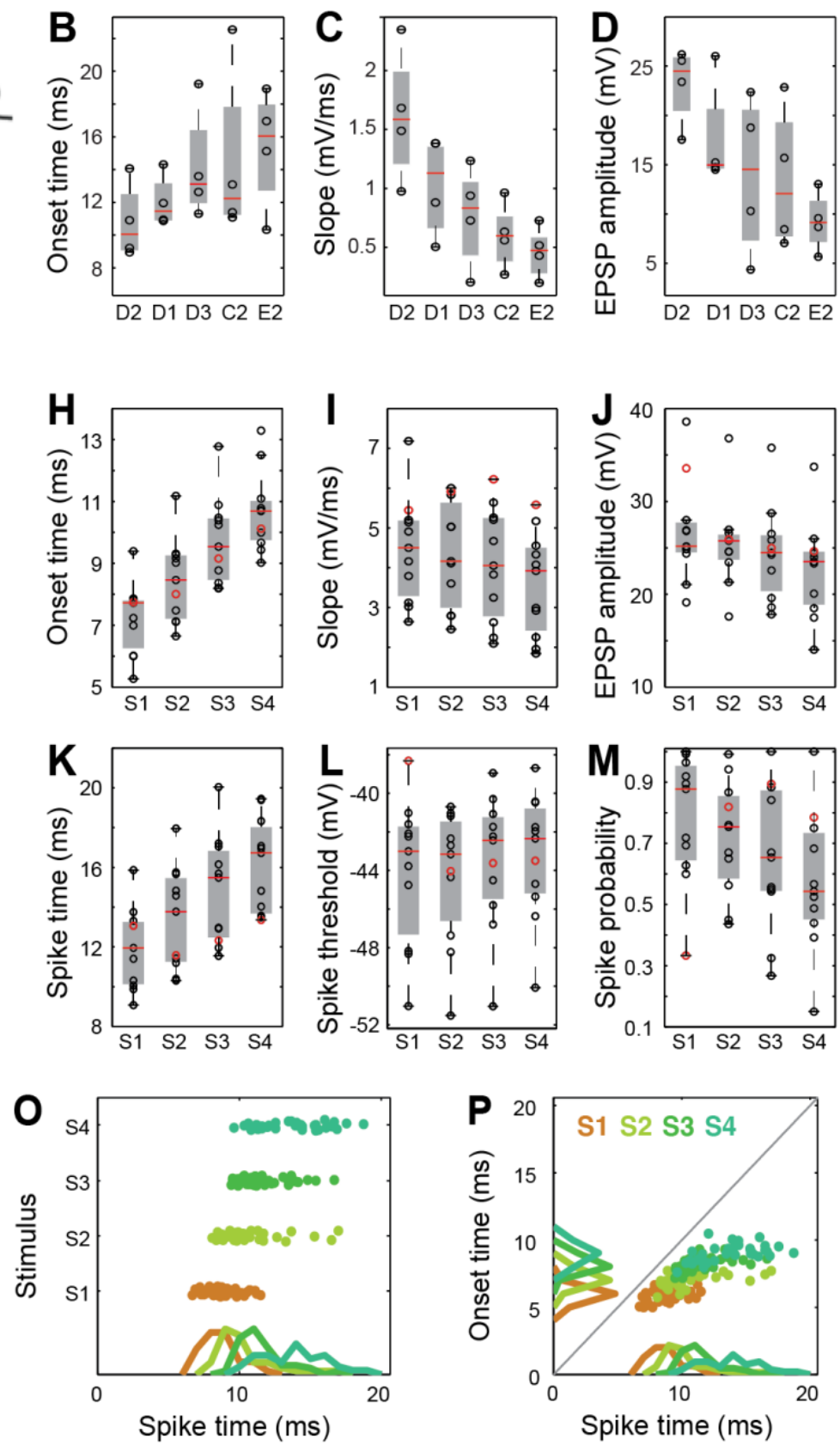
Figure 1. In vivo and in vitro stimulus representation in single L2/3 somatosensory cortical neurons

691 (A) We deflected whisker D2 and its first order neighbors (D1,D3,C2,E2) individually to determine the 692 spatial encoding properties of cortical L2/3 pyramidal neurons in the D2 barrel under anesthesia using 693 whole cell current clamp recordings. (B-D) EPSP response to in vivo stimulation. Analysis of the EPSP 694 parameters showed that principal whisker stimulation was correlated with earlier onset times (B), larger 695 slopes (C) and larger amplitudes (D) compared to the surround whiskers. Onset time was described as the 696 latency between stimulus onset and the time it takes for the membrane to reach $10 \%$ of the peak somatic 697 EPSP amplitude. The EPSP slope was calculated to be between 10-90\% of the somatic EPSP. The amplitude was measured at the peak. All measurements were performed on monosynaptic EPSPs. (E-M) Response to in vitro stimulation mimicking in vivo stimulation. Due to the sparse nature of action potentials in vivo, we developed a stimulation protocol to mimic the subthreshold stimulus encoding properties of L2/3 neurons in vitro. (E) Whole cell intracellular current clamp recordings were performed

702 in L2/3 while L4 neurons were stimulated using a bipolar electrode. (F) Soma location of randomly selected

703 neurons. (G) The stimuli were direct current injections with equal maximal amplitudes as the in vivo 704 EPSCs, but the rising slope of the current was systematically reduced across the four stimulus conditions 705 (see (Huang et al., 2016)). (H-M) L2/3 pyramidal neurons' responses to L4 stimulation. Each circle shows 706 the average (over trials) response of one neuron ( $\mathrm{N}=11)$. (H-J) EPSP response to in vitro stimulation. $\mathbf{H}$ : 707 Onset time, I: Slope, J: EPSP amplitude; (K-M) Spike response to in vitro stimulation. K: Spike time, i.e. 708 latency to spike after stimulus onset; L: Spike threshold, described as the membrane potential at which the 709 second derivative reaches a global (positive) maximum; M: Action potential (i.e. spike) probability, across 710 trials. (N-P) Spike versus EPSP response to in vitro stimulus. While both EPSP and spike parameters 711 displayed an average dependence on the stimulus, EPSP parameters are more accurately determined by the 712 stimulus than spike parameters on single trials. 

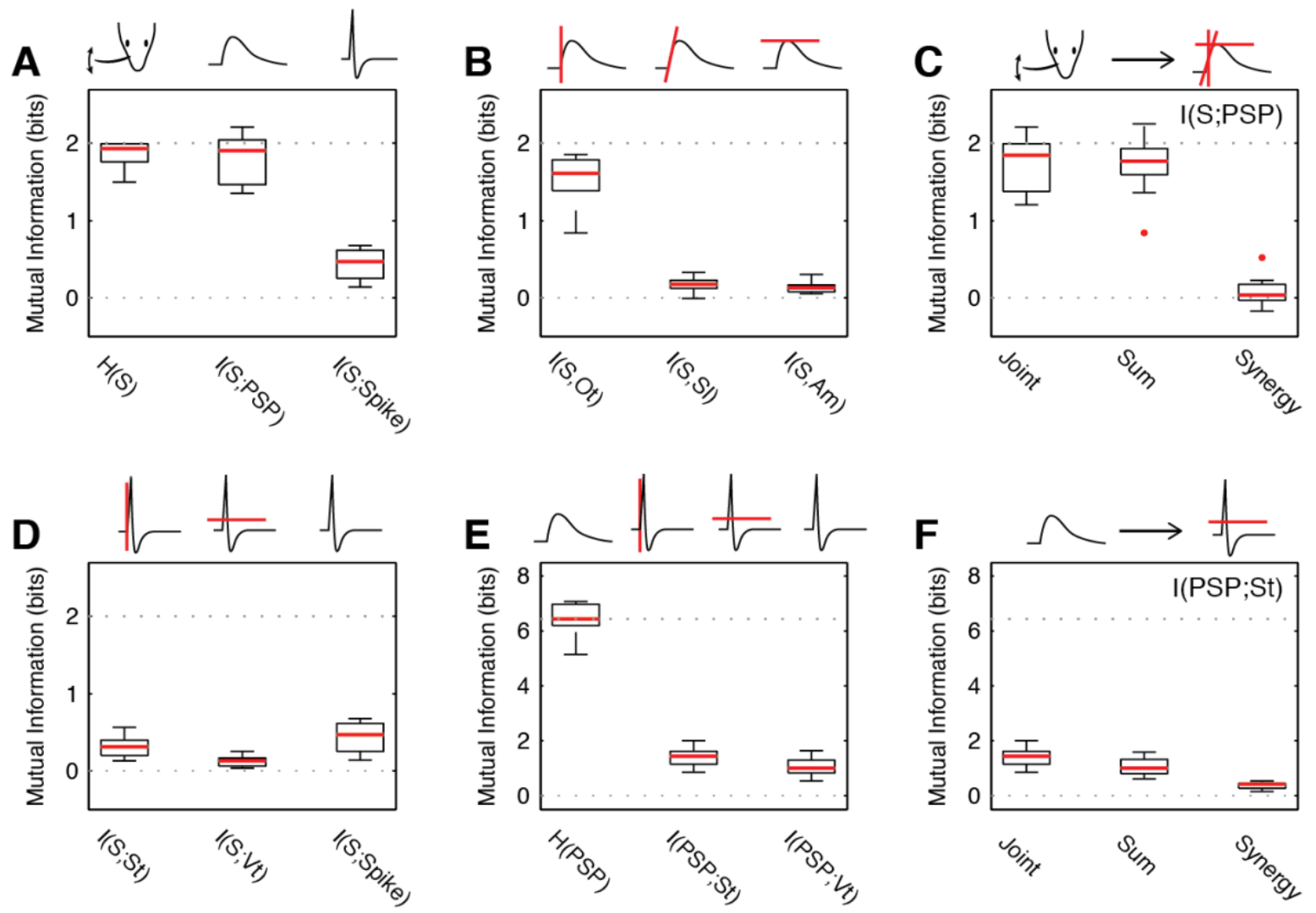

Figure 2. Postsynaptic potentials encode substantially more stimulus information than spikes in vitro

716 (A) The information between PSPs and the stimulus is significantly higher than the information between

717 spikes and the stimulus. While the PSP contains a large fraction of the stimulus entropy (95\%, I(S;PSP),

$7181.81 \pm 0.31$ bit vs. $\mathrm{H}(\mathrm{S}), 1.86 \pm 0.17$ bit, $\mathrm{p}=0.16$ ), most of this information is not transferred to the spike

719 (I(S;Spike), $0.47 \pm 0.19$ bit, 24\%). (B) The majority of the information in the PSP is carried by the onset

720 timing (Ot, $1.6 \pm 0.31$ bit, 85\%), while slope (S1, $0.17 \pm 0.09$ bit, 10\%) amplitude (Am, $0.13 \pm 0.07$ bit, 5\%)

721 carry only small amounts of information. (C) Ot, Sl, and Am add their information independently, as the

722 synergy between them is close to 0 (Synergy : $0.03 \pm 0.18$ bit, $\mathrm{p}=0.15$, $\mathrm{t}$-test). (D) The information in the

723 spike is contributed by spike time ( $\mathrm{St}, 0.31 \pm 0.13 \mathrm{bit}, 16 \%$ ) and threshold (Vt, $0.12 \pm 0.08$ bit, $6.2 \%$ ), and

724 jointly only reach $21 \%$ of the total information (repeated from A). (E) Substantial information transfer 725 occurs between the PSP and the spike, although this constitutes only $22 \%(\mathrm{St})$ and $15 \%(\mathrm{Vt})$ of the entropy 
726 in the PSP. (F) The information in the properties of the PSP adds largely independently to the joint 727 information, with a small but highly significant synergistic contribution of different PSP properties ( 0.41

$728 \pm 0.13$ bit, $\left.6.4 \%, \mathrm{p}<10^{-5}\right)$. In all figures data is plotted as inter-quartile intervals and red lines denote the 729 median of each distribution. Outliers are plotted as red dots. The dotted line denotes the maximal stimulus 730 entropy. 

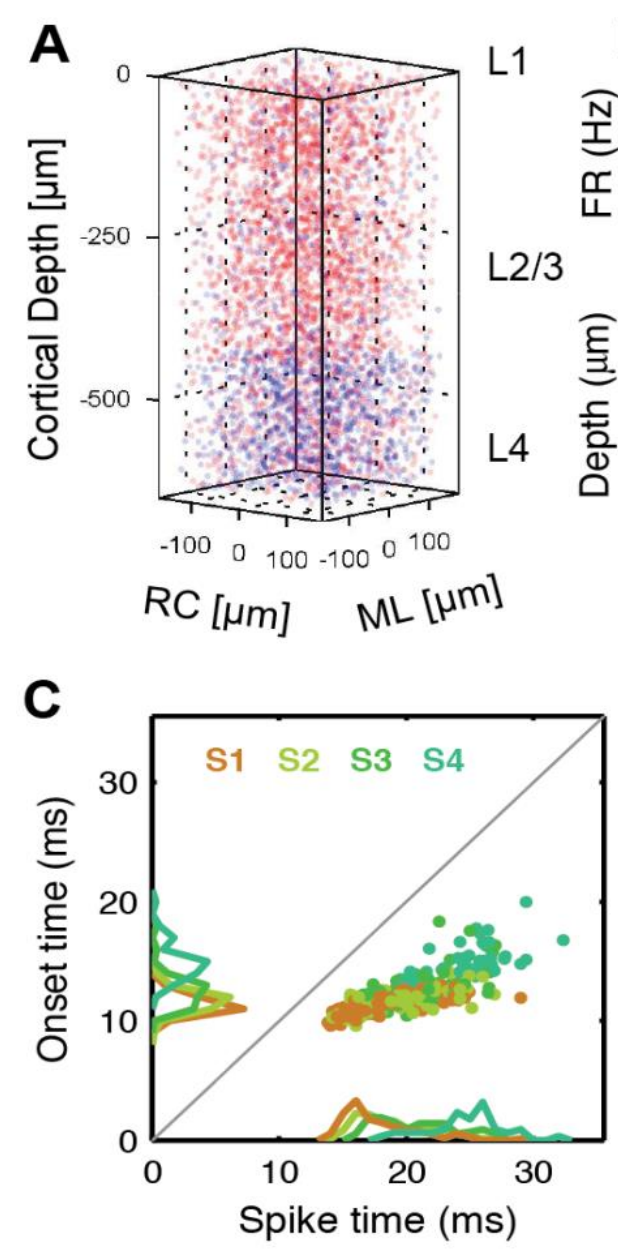
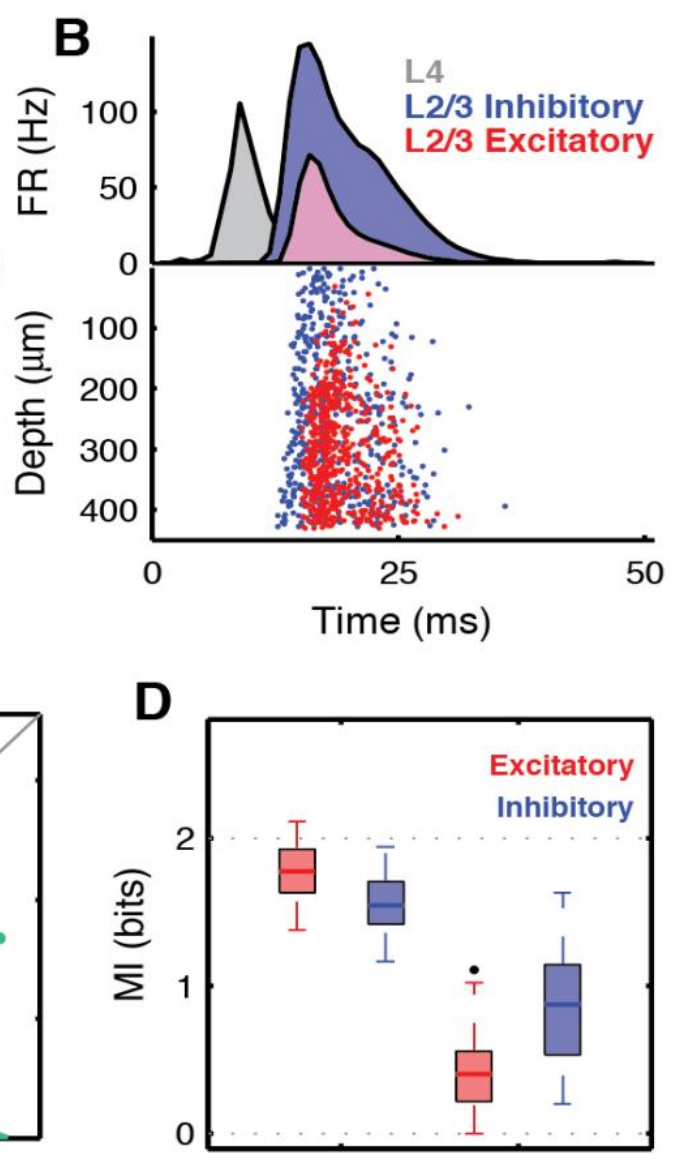

I(S;PSP) I(S;Spike)

Figure 3. Anatomically constrained barrel column in silico reproduces the relationships between sub-

\section{4 and supra-threshold information}

735 (A) An anatomically based model of a barrel column for L2/3/4 was generated to analyze the information

736 transfer between L4 and L2/3 in analogy to the physiological recordings (see Huang et al. (2020) for

737 details). (B) In response to stimulation in L4 with a whisker-like PSTH (grey), excitatory (red) and

738 inhibitory (blue) cells respond in L2/3, with inhibitory activity eventually extinguishing the total activity in

739 the network. (C) Corresponding to the in vitro/in vivo data, the timing of PSPs for a given stimulus is more 
740 precise than the spikes they evoke (compare to Fig. 1L). (D) The relationship between PSPs and spikes in

741 terms of timing and reliability leads to single cell mutual information very similar to the recorded data

742 (excitatory cells, compare to Fig.2A). Inhibitory cells (not recorded), show less information in their PSP

743 response, but more information in the spikes (all properties combined for both cell-types). Dotted line:

744 stimulus entropy. 


\section{A Population Information Analysis}

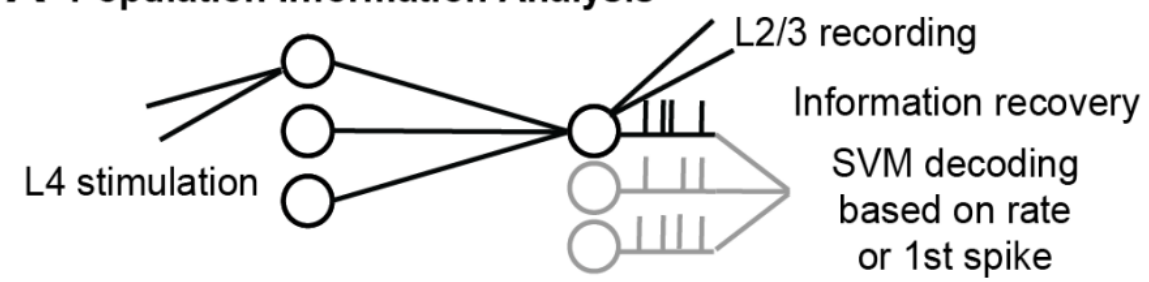

B

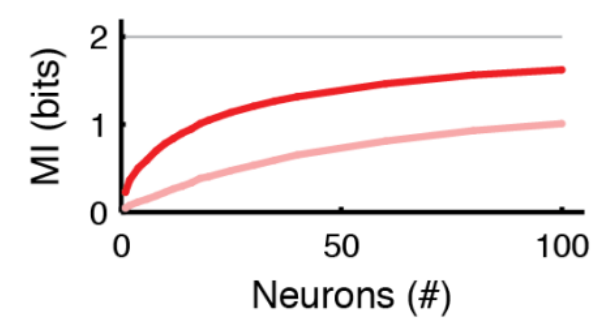

Validity

C

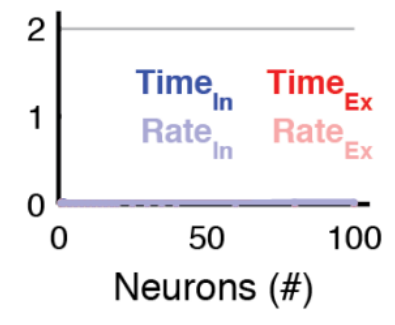

\section{L4 Activity}
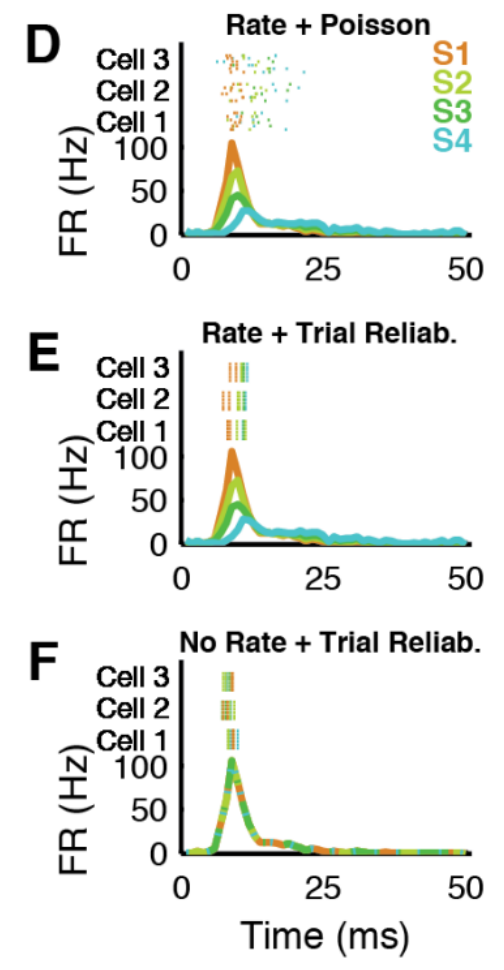

L2/3 Decoding
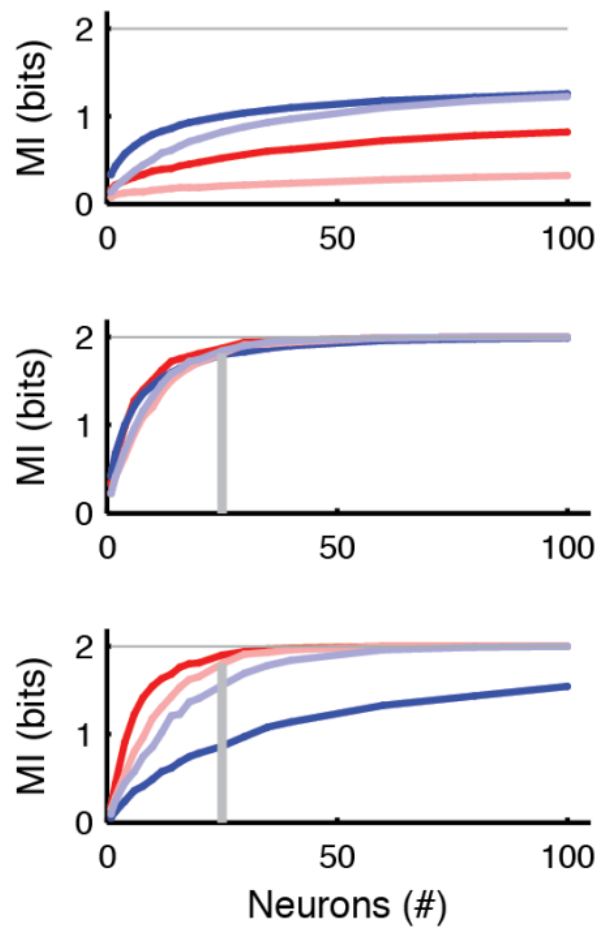
Figure 4. Information recovery in neural populations recorded in vitro.

747 If a postsynaptic EPSP carries near complete information about the stimulus in the periphery 748 (Figure 2), how does the postsynaptic neuron reconstruct this information from poorly informative 749 action potentials of the presynaptic neurons? (A) To address this question we evaluate the mutual 750 information from population spike trains of groups of excitatory or inhibitory neurons. To prevent 751 the sampling bias, MI is estimated between the stimulus and an SVM decoding from the population

752 response. (B) Population information estimated from bootstrapped in vitro recordings show nearly 753 complete recovery of stimulus information. Asymptote is reached above $81 \%$ for 100 neurons for 754 temporal decoding (dark red), and remains systematically lower for the rate-based decoding (light 755 red). (C) Estimating population information for non-informative stimuli (identical PSTH, Poisson756 spiking) leads to vanishingly low MI values, demonstrating that the analysis does not introduce a 757 positive bias. (D) If the population activity in L4 is only constrained by the PSTH and otherwise 758 spikes are drawn according to Poisson-distributions (bottom left, different colors = different 759 stimuli), then inhibitory neurons carry more information for both time (dark blue) and rate (light 760 blue) decoding, than excitatory neurons (dark \& light red respectively). The gray line denotes the 761 entropy of the stimulus. (E) If PSTHs differ across stimuli but spike timing is stereotypical across 762 trials ('Rate + Trial Reliability', top left, multiple trials per neuron above each other), coding 763 becomes highly effective and independent of the cell-type and coding strategy ( 25 cells). (F) If 764 L4 PSTHs do not distinguish stimuli, but only the timing of individual neurons across trials is 765 stereotypical (No Rate + Trial Reliability, top left), a remarkable shift occurs, with excitatory 766 neurons reaching almost complete information for much smaller group sizes ( 25 cells). In all 767 plots the vertical grey line indicates where $90 \%$ of the information is represented. 
bioRxiv preprint doi: $\mathrm{https}$ //doi.org/10.1101/2020.1208.415729. this version posted December 15,2020 . The copyright holder for this preprint (which was not certified by peer review) is the author/funder, who has granted bioRxiv a license to display the preprint in perpetuity. It is made available under aCC-BY-ND 4.0 International license.

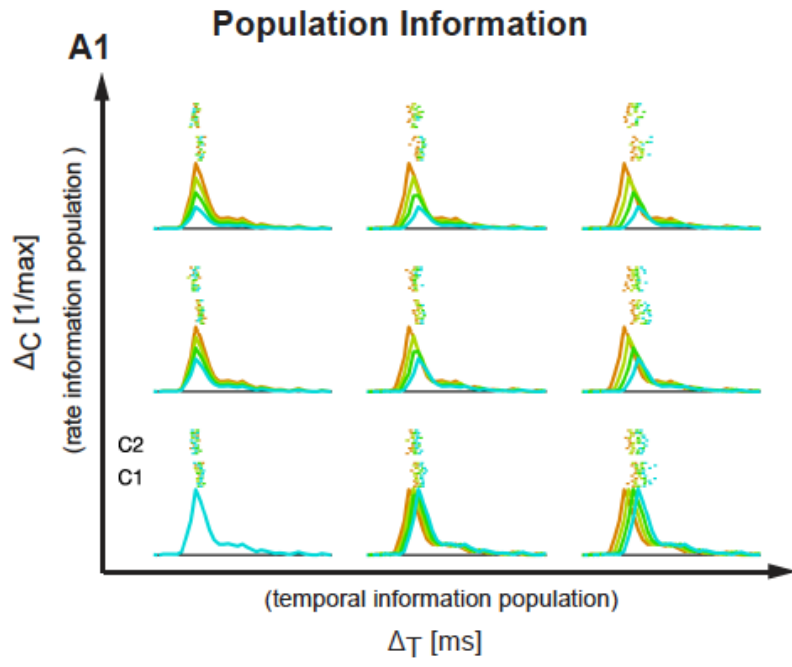

A2

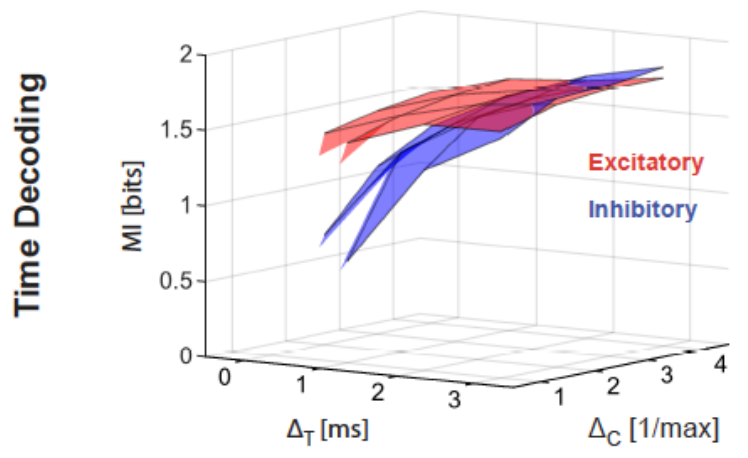

A3

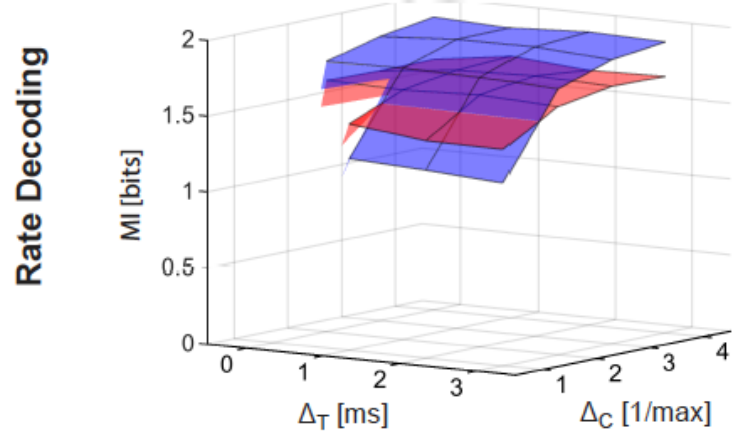

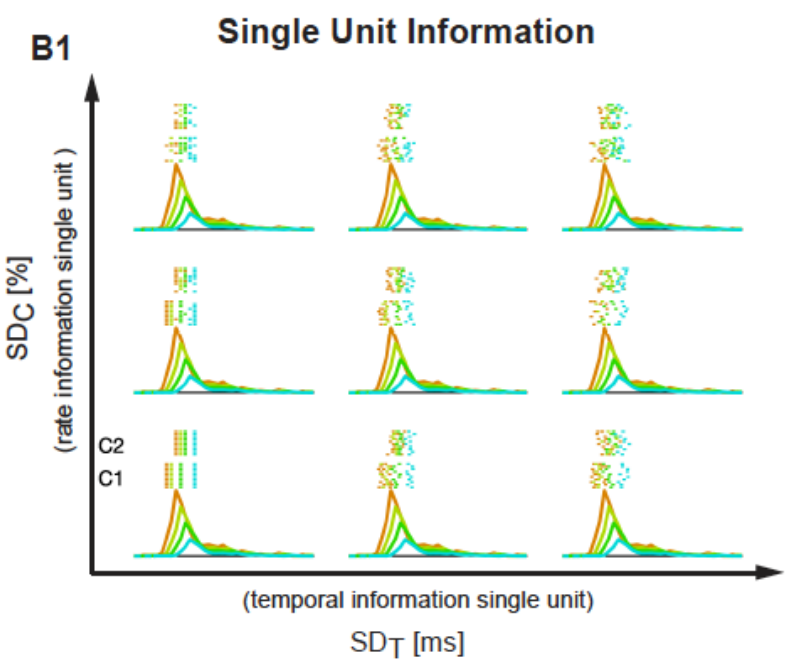

B3

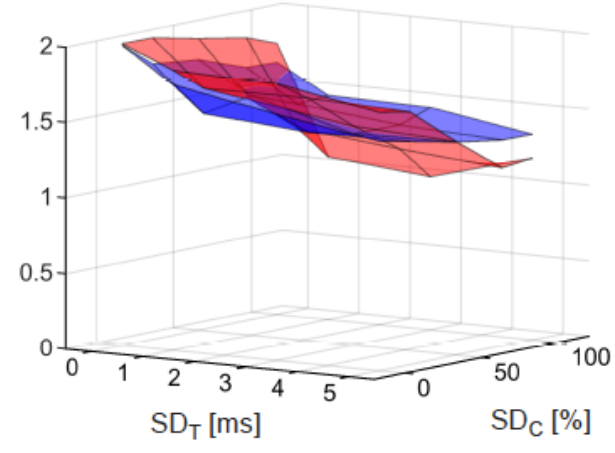

B2

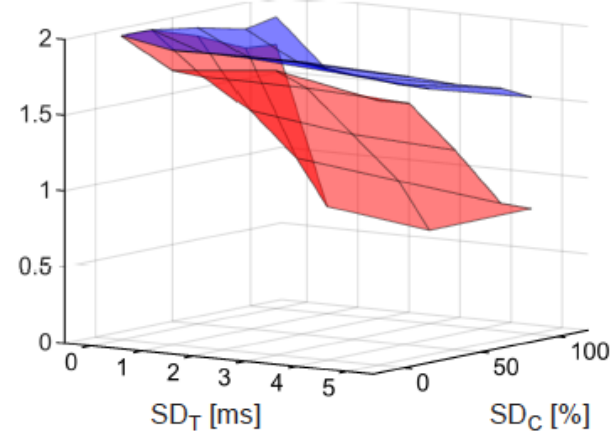


770 Figure 5. Stimulus encoding by presynaptic single neurons and populations of neurons selectively

771 influences the decoding performance of the postsynaptic excitatory or inhibitory neurons, 772 respectively.

773 (A1) Stimulus information can be encoded in differences in rate or timing on the level of the 774 population PSTH. Different combinations of these two coding dimensions are varied, with $\Delta_{\mathrm{T}}$ 775 (abscissa) indicating different timing for different stimuli (different colors, see Fig. 4), and $\Delta_{\mathrm{C}}$ 776 (ordinate) indicating different rates for different stimuli. Maximal information is achieved for high 777 values of $\Delta_{\mathrm{T}}$ and $\Delta \mathrm{c}$. For each condition the population PSTHs and two example cells are shown 778 (raster plot for 10 trials, above). Spike-times of individual neurons are Poisson-distributed given 779 the PSTH. NB C1 and C2 denote the responses of two different example cells. (A2) Decoding of 780 first spike timing reveals a greater sensitivity of inhibitory neurons (blue) to the level of 781 information in the L4 population response, both for time and rate information in L4. Conversely, 782 excitatory neurons (red) are comparatively insensitive. (A3) Decoding of rate again reveals a 783 greater sensitivity of inhibitory neurons to the level of information in the L4 response for different 784 rates. Since we did not limit the time window of analysis, neither of the cell types is influenced by 785 variation in time, while leaving the rate information unchanged. (B1) Stimulus information can 786 also be encoded in the reliable discharge of single units. We modulated the reliability by 787 introducing variability in timing (SDT) or variability in count (SDc) independent of each other.

788 Maximal information is achieved for $\mathrm{SD}_{\mathrm{T}}$ and $\mathrm{SD}_{\mathrm{C}}$ both close to 0, i.e. perfectly reliable responses. 789 Colors and raster plots as in A1. (B2) Decoding of first spike timing reveals a great sensitivity of 790 excitatory neurons to the $\mathrm{L} 4$ information in single unit responses for both variability in time (SDT) 791 and rate $\left(\mathrm{SD}_{\mathrm{C}}\right)$. (B3) Decoding of rate shows a very strong sensitivity of excitatory neurons on the 792 single unit information. Conversely, inhibitory neurons exhibit almost no sensitivity to single unit 793 information in L4, and are thus dominated by L4 population information. 

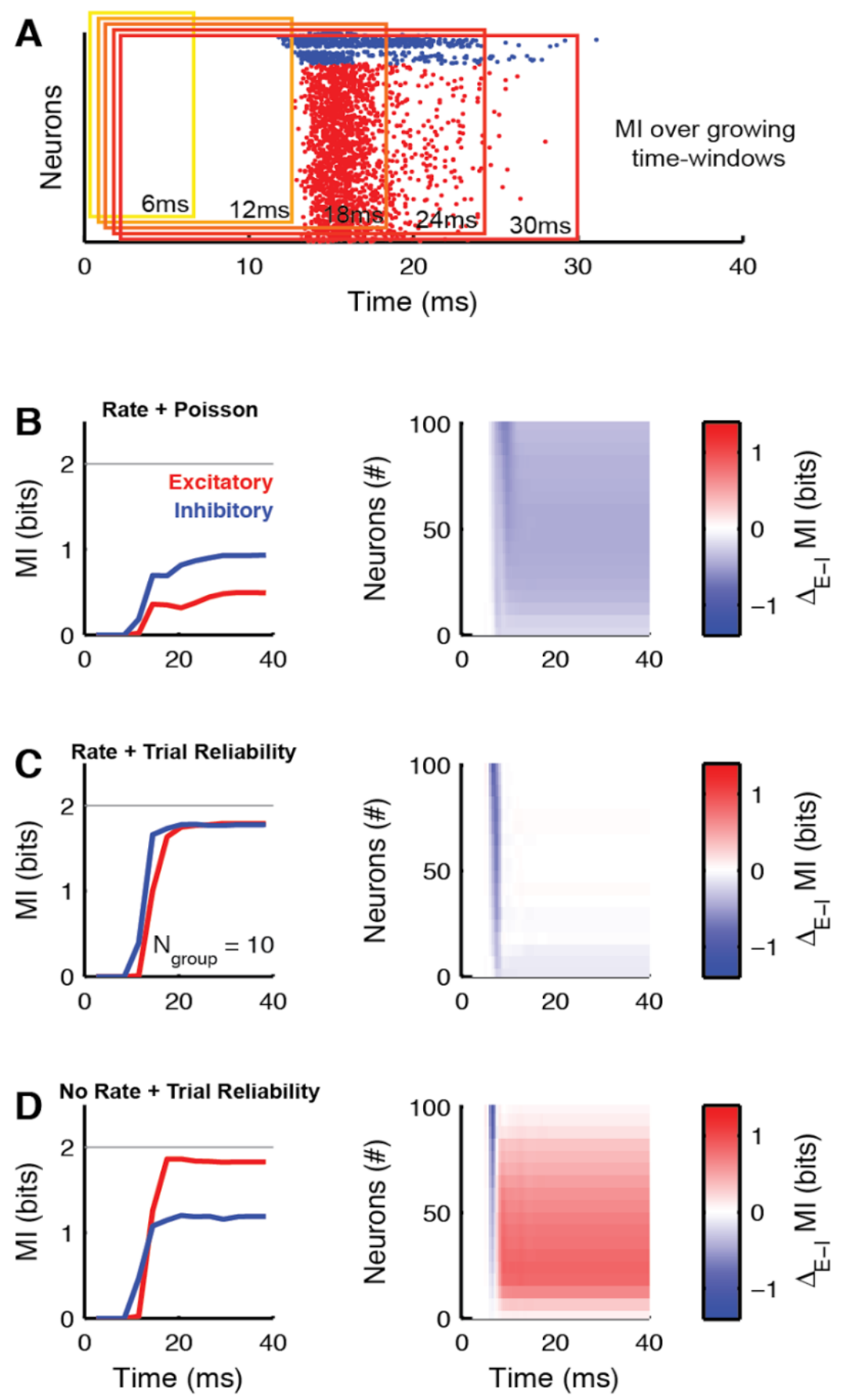


\section{Figure 6. Neuronal information recovery is completed in <20ms after stimulus onset.}

796 (A) Time-scales of information recovery computed by calculating the MI between stimulus (not shown)

797 and spike trains (single trial example shown) over time-windows of increasing lengths (6-30ms at 3ms

798 steps) (B) For the 'Rate + Poisson' encoding in L4, both excitatory (red) and inhibitory (blue) neurons in

799 L2/3 reach their respective maximal information (left: group size of 10 cells, the gray line denotes the

800 entropy of the stimulus), $\sim 25-30 \mathrm{~ms}$ after stimulus onset. L2/3 inhibitory neurons (blue) encode more

801 information, independent from the peri-stimulus time and group size (right). The color code shows the

802 difference in MI between the excitatory and inhibitory groups, with red for larger MI in the excitatory, and

803 blue for greater MI for the inhibitory neurons. (C) For the 'Rate + Trial Reliability' condition in L4, the

804 information content of the two populations is quite similar (left) with a slight advantage for the inhibitory

805 neurons at early times, but no dependence on group size (right). (D) In the "No Rate - Trial Reliability",

806 case, the divergence between information content only begins around $12 \mathrm{~ms}$ after stimulus onset, after which

807 excitatory neurons achieve a substantial coding advantage, especially for smaller group sizes. 
A Speculated coding schemas for touch

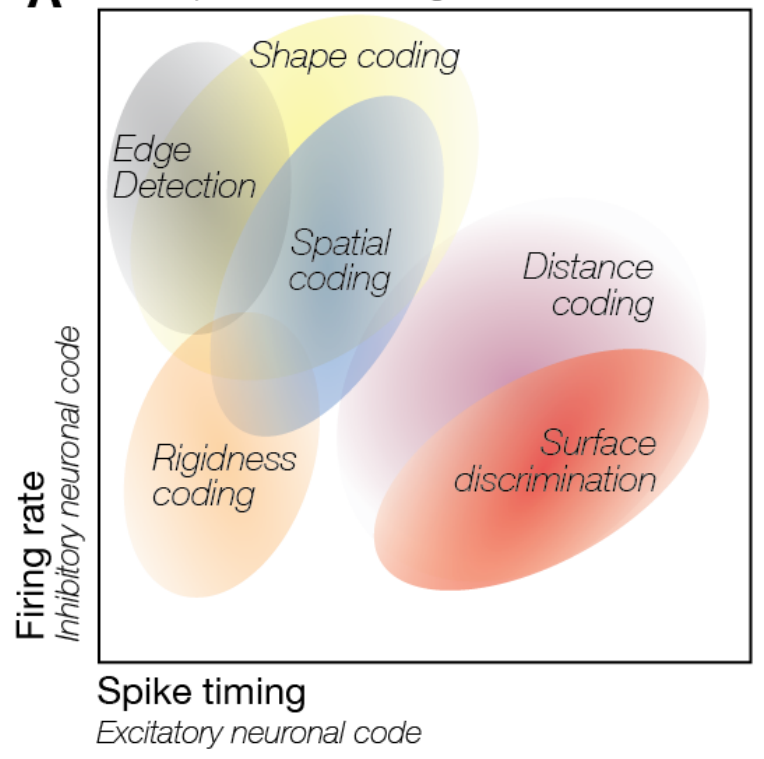

B

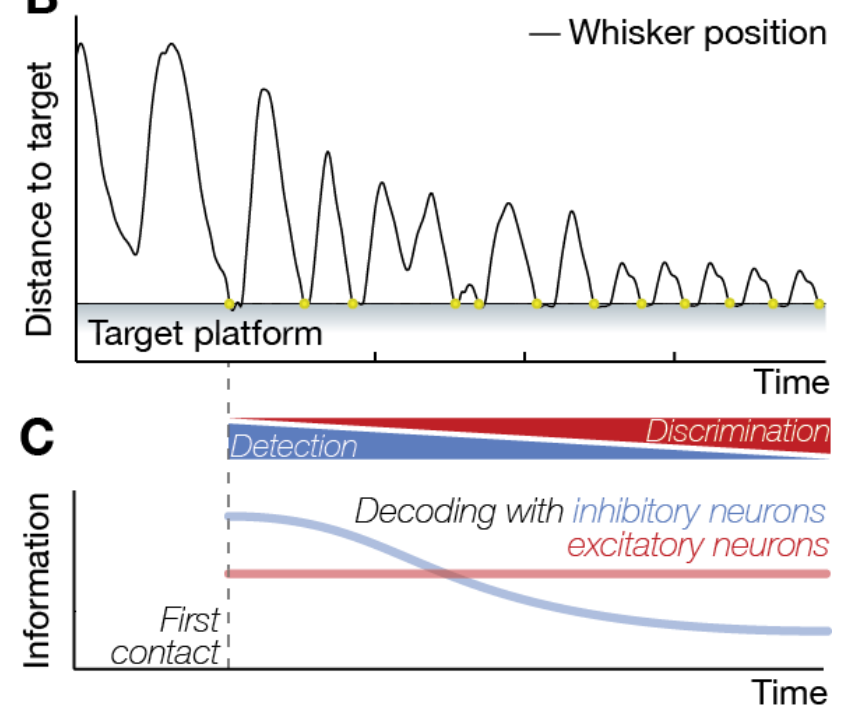

Figure 7. Multiplexed coding of touch.

810 If intracellular information transfer, i.e. from EPSP-to-spike, results in a significant loss of near complete 811 information, originally available in a single EPSP (Fig. 2), and if this information is recovered in local 812 networks (Fig. 4 and 5) before the next sensory stimulus arrives (Fig. 6) using the rate and timing of spikes 813 at the single cell and population levels (Fig. 4-6), selective decoding of stimulus properties by excitatory 814 and inhibitory neural populations (Fig.5) will result in a multiplexed code for sensory processing. (A) If 815 excitatory and inhibitory neurons preferentially decode the stimulus information from the spike timing of 816 individual neurons and the population rate of presynaptic neuronal activity (Fig. 5), respectively, 817 information content of the activity in excitatory and inhibitory neurons should vary predictably - see the 818 suggested coding schema for touch. (B-C) The information content across the neural populations will also 819 vary depending on the complexity of the stimulus. (B) During tactile object localization in freely behaving 820 animals (Celikel and Sakmann, 2007; Voigts et al., 2015, 2008), for example, as the animal approaches the 821 tactile target and makes multiple contacts, the information content will change not only because the 822 kinematics of touch varies, e.g. the amplitude whisker deflections is reduced to match the predicted position 823 of the sensory target (Voigts et al., 2015), but also the neurons will represent different features of the sensory 
824 target. (C) We speculate that information in the inhibitory neurons will better predict the stimulus location,

825 although the information content of the excitatory neurons will eventually supersede as surface features are

826 encoded with the subsequent contacts with the target.

827

828 


\section{References}

830 Alenda A, Molano-Mazón M, Panzeri S, Maravall M. 2010. Sensory input drives multiple intracellular information streams in somatosensory cortex. The Journal of Neuroscience 30:10872-84. doi:10.1523/JNEUROSCI.6174-09.2010

Allen CB, Celikel T, Feldman DE. 2003. Long-term depression induced by sensory deprivation during cortical map plasticity in vivo. Nature neuroscience 6:291-9. doi:10.1038/nn1012

Amarasingham A, Chen T-L, Geman S, Harrison MT, Sheinberg DL. 2006. Spike Count Reliability and the Poisson Hypothesis. J Neurosci 26:801-809. doi:10.1523/JNEUROSCI.2948-05.2006

Amarasingham A, Harrison MT, Hatsopoulos NG, Geman S. 2011. Conditional modeling and the jitter method of spike resampling. Journal of Neurophysiology 107:517-531. doi:10.1152/jn.00633.2011

Arabzadeh E, Zorzin E, Diamond ME. 2005. Neuronal Encoding of Texture in the Whisker Sensory Pathway. PLOS Biology 3:e17. doi:10.1371/journal.pbio.0030017

Avermann M, Tomm C, Mateo C, Gerstner W, Petersen CCH. 2012. Microcircuits of excitatory and inhibitory neurons in layer $2 / 3$ of mouse barrel cortex. Journal of Neurophysiology 107:3116-3134. doi:10.1152/jn.00917.2011

Azarfar A, Calcini N, Huang C, Zeldenrust F, Celikel T. 2018. Neural coding: A single neuron's perspective. Neuroscience \& Biobehavioral Reviews 94:238-247. doi:10.1016/j.neubiorev.2018.09.007

Barth AL, Poulet JFA. 2012. Experimental evidence for sparse firing in the neocortex. Trends in Neurosciences 35:345-355. doi:10.1016/j.tins.2012.03.008

Bialek W, Rieke F. 1992. Reliability and information transmission in spiking neurons. Trends in Neurosciences 15:428-434. doi:10.1016/0166-2236(92)90005-S

Bialek W, Rieke F, de Ruyter van Steveninck RR, Warland D. 1991. Reading a Neural Code. Science 252:1854-1857.

Billings G, Piasini E, Lorincz A, Nusser Z, Silver RA. 2014. Network Structure within the Cerebellar Input Layer Enables Lossless Sparse Encoding. Neuron 83:960-974. doi:10.1016/j.neuron.2014.07.020

Blatow M, Rozov A, Katona I, Hormuzdi SG, Meyer AH, Whittington MA, Caputi A, Monyer H. 2003. A Novel Network of Multipolar Bursting Interneurons Generates Theta Frequency Oscillations in Neocortex. Neuron 38:805-817. doi:10.1016/S08966273(03)00300-3

Brecht M, Roth A, Sakmann B. 2003. Dynamic receptive fields of reconstructed pyramidal cells in layers 3 and 2 of rat somatosensory barrel cortex. The Journal of physiology 553:24365. doi:10.1113/jphysiol.2003.044222

Brecht M, Schneider M, Sakmann B, Margrie TW. 2004. Whisker movements evoked by stimulation of single pyramidal cells in rat motor cortex. Nature 427:704-710. 
doi:10.1038/nature02266

Brette R. 2015. Philosophy of the spike: rate-based vs. spike-based theories of the brain.

Frontiers in Systems Neuroscience 9. doi:10.3389/fnsys.2015.00151

Caputi A, Rozov A, Blatow M, Monyer H. 2009. Two calretinin-positive GABAergic cell types in layer $2 / 3$ of the mouse neocortex provide different forms of inhibition. Cereb Cortex 19:1345-1359. doi:10.1093/cercor/bhn175

Celikel T, Sakmann B. 2007. Sensory integration across space and in time for decision making in the somatosensory system of rodents. Proceedings of the National Academy of Sciences 104:1395-400. doi:10.1073/pnas.0610267104

Celikel T, Szostak VA, Feldman DE. 2004. Modulation of spike timing by sensory deprivation during induction of cortical map plasticity. Nature neuroscience 7:534-541. doi:10.1038/nn1222

Chklovskii DB, Koulakov AA. 2004. MAPS IN THE BRAIN: What Can We Learn from Them? Annual Review of Neuroscience 27:369-392. doi:10.1146/annurev.neuro.27.070203.144226

Clem RL, Celikel T, Barth AL. 2008. Ongoing in vivo experience triggers synaptic metaplasticity in the neocortex. Science 319:101-4. doi:10.1126/science.1143808

Crochet S, Petersen CCH. 2006. Correlating whisker behavior with membrane potential in barrel cortex of awake mice. Nature Neuroscience 9:608-610. doi:10.1038/nn1690

Crochet S, Poulet JFA, Kremer Y, Petersen CCH. 2011. Synaptic Mechanisms Underlying Sparse Coding of Active Touch. Neuron 69:1160-1175. doi:10.1016/j.neuron.2011.02.022

Dalgleish HWP, Russell LE, Packer AM, Roth A, Gauld OM, Greenstreet F, Thompson EJ, Häusser M. 2020. How many neurons are sufficient for perception of cortical activity? eLife 9:e58889. doi:10.7554/eLife.58889

De Kock CPJ, Bruno RM, Spors H, Sakmann B. 2007. Layer- and cell-type-specific suprathreshold stimulus representation in rat primary somatosensory cortex. The Journal of Physiology 581:139-154. doi:10.1113/jphysiol.2006.124321

DeCharms RC, Zador AM. 2000. Neural representation and the cortical code. Annual review of neuroscience 23:613-647.

Denève S. 2008. Bayesian spiking neurons I: inference. Neural Computation 20:91-117. doi:10.1162/neco.2008.20.1.91

Denève S, Machens CK. 2016. Efficient codes and balanced networks. Nature neuroscience 19:375-82. doi:10.1038/nn.4243

Denève S, Pouget A. 2003. Basis functions for object-centered representations. Neuron 37:34759.

Destexhe A, Rudolph M, Paré D. 2003. The high-conductance state of neocortical neurons in vivo. Nature Reviews Neuroscience 4:739-51. doi:10.1038/nrn1198

Diamond ME, Petersen RS, Harris JA. 1999. Learning through maps: functional significance of 
topographic organization in primary sensory cortex. J Neurobiol 41:64-68.

Doron G, von Heimendahl M, Schlattmann P, Houweling AR, Brecht M. 2014. Spiking Irregularity and Frequency Modulate the Behavioral Report of Single-Neuron Stimulation. Neuron 81:653-663. doi:10.1016/j.neuron.2013.11.032

Eliasmith C, Anderson CH. 2002. Of neurons and engineersNeural Engineering. Computation, Representation, and Dynamics in Neurobiological Systems. Cambridge, Massachusets, London, England: MIT Press. pp. 1-23.

Feldmeyer D, Lübke J, Sakmann B. 2006. Efficacy and connectivity of intracolumnar pairs of layer 2/3 pyramidal cells in the barrel cortex of juvenile rats: Layer 2/3 pyramidal cell synapses. The Journal of Physiology 575:583-602. doi:10.1113/jphysiol.2006.105106

Feldmeyer D, Lübke J, Silver RA, Sakmann B. 2002. Synaptic connections between layer 4 spiny neurone- layer $2 / 3$ pyramidal cell pairs in juvenile rat barrel cortex: physiology and anatomy of interlaminar signalling within a cortical column. The Journal of Physiology 538:803. doi:10.1113/jphysiol.2001.012959

Finnerty GT, Connors BW. 2000. Sensory deprivation without competition yields modest alterations of short-term synaptic dynamics. PNAS 97:12864-12868. doi:10.1073/pnas.230175697

Franke F, Fiscella M, Sevelev M, Roska B, Hierlemann A, Azaredo da Silveira R. 2016. Structures of Neural Correlation and How They Favor Coding. Neuron 89:409-422. doi:10.1016/j.neuron.2015.12.037

Gao P, Ganguli S. 2015. On simplicity and complexity in the brave new world of large-scale neuroscience. Current Opinion in Neurobiology 32:148-155. doi:10.1016/j.conb.2015.04.003

Gollisch T, Meister M. 2008. Rapid neural coding in the retina with relative spike latencies. Science 319:1108-1111. doi:10.1126/science.1149639

Harding-Forrester S, Feldman DE. 2018. Chapter 4 - Somatosensory maps In: Vallar G, Coslett HB, editors. Handbook of Clinical Neurology, The Parietal Lobe. Elsevier. pp. 73-102. doi:10.1016/B978-0-444-63622-5.00004-8

Harris KD, Mrsic-Flogel TD. 2013. Cortical connectivity and sensory coding. Nature 503:51-8. doi:10.1038/nature12654

Helmstaedter M, Staiger JF, Sakmann B, Feldmeyer D. 2008. Efficient Recruitment of Layer 2/3 Interneurons by Layer 4 Input in Single Columns of Rat Somatosensory Cortex. Journal of Neuroscience 28:8273-8284. doi:10.1523/JNEUROSCI.5701-07.2008

Holmgren C, Harkany T, Svennenfors B, Zilberter Y. 2003. Pyramidal cell communication within local networks in layer 2/3 of rat neocortex. The Journal of Physiology 551:139153. doi:10.1113/jphysiol.2003.044784

Houweling AR, Brecht M. 2008. Behavioural report of single neuron stimulation in somatosensory cortex. Nature 451:65-68. doi:10.1038/nature06447

Huang C, Resnik A, Celikel T, Englitz B. 2016. Adaptive Spike Threshold Enables Robust and 
Temporally Precise Neuronal Encoding. PLoS Computational Biology 12. doi:10.1371/journal.pcbi.1004984

Huang C, Zeldenrust F, Celikel T. 2020. Cortical representation of touch in silico. bioRxiv. doi:https://doi.org/10.1101/371252

Ince RAA, Mazzoni A, Petersen RS, Panzeri S. 2010a. Open source tools for the information theoretic analysis of neural data. Front Neurosci 3. doi:10.3389/neuro.01.011.2010

Ince RAA, Panzeri S, Kayser C. 2013. Neural Codes Formed by Small and Temporally Precise Populations in Auditory Cortex. J Neurosci 33:18277-18287. doi:10.1523/JNEUROSCI.2631-13.2013

Ince RAA, Senatore R, Arabzadeh E, Montani F, Diamond ME, Panzeri S. 2010b. Informationtheoretic methods for studying population codes. Neural Networks 23:713-727. doi:10.1016/j.neunet.2010.05.008

Izhikevich EM. 2004. Which model to use for cortical spiking neurons? IEEE Transactions on Neural Networks 15:1063-1070.

Izhikevich EM. 2003. Simple Model of Spiking Neurons. IEEE Transactions on Neural Networks 14:1572-1596. doi:10.1109/TNN.2003.820440

Johansson RS, Birznieks I. 2004. First spikes in ensembles of human tactile afferents code complex spatial fingertip events. Nature Neuroscience 7:170-7. doi:10.1038/nn1177

Kaas JH. 1997. Topographic Maps are Fundamental to Sensory Processing. Brain Research Bulletin 44:107-112. doi:10.1016/S0361-9230(97)00094-4

Kayser C, Ince RAA, Panzeri S. 2012. Analysis of Slow (Theta) Oscillations as a Potential Temporal Reference Frame for Information Coding in Sensory Cortices. PLOS Computational Biology 8:e1002717. doi:10.1371/journal.pcbi.1002717

Kayser C, Logothetis NK, Panzeri S. 2010. Millisecond encoding precision of auditory cortex neurons. PNAS 107:16976-16981. doi:10.1073/pnas.1012656107

Knudsen EI, Lac S, Esterly SD. 1987. Computational Maps in the Brain. Annual Review of Neuroscience 10:41-65. doi:10.1146/annurev.ne.10.030187.000353

Ko H, Hofer SB, Pichler B, Buchanan K a, Sjöström PJ, Mrsic-Flogel TD. 2011. Functional specificity of local synaptic connections in neocortical networks. Nature 473:87-91. doi:10.1038/nature09880

Kole K, Scheenen W, Tiesinga P, Celikel T. 2018. Cellular diversity of the somatosensory cortical map plasticity. Neuroscience \& Biobehavioral Reviews 84:100-115. doi:10.1016/j.neubiorev.2017.11.015

Li N, Chen T-W, Guo ZV, Gerfen CR, Svoboda K. 2015. A motor cortex circuit for motor planning and movement. Nature 519:51-56. doi:10.1038/nature14178

Litwin-Kumar A, Doiron B. 2012. Slow dynamics and high variability in balanced cortical networks with clustered connections. Nature neuroscience 15:1498-505. doi:10.1038/nn.3220

London M, Roth A, Beeren L, Häusser M, Latham PE. 2010. Sensitivity to perturbations in vivo 
984

985

986

987

988

989

990

991

992

993

994

995

996

997

998

999

1000

1001

1002

1003

1004

1005

1006

1007

1008

1009

1010

1011

1012

1013

1014

1015

1016

1017

1018

1019

1020

1021

1022

implies high noise and suggests rate coding in cortex. Nature 466:123-127. doi:10.1038/nature09086

Lübke J, Roth A, Feldmeyer D, Sakmann B. 2003. Morphometric Analysis of the Columnar Innervation Domain of Neurons Connecting Layer 4 and Layer 2/3 of Juvenile Rat Barrel Cortex. Cereb Cortex 13:1051-1063. doi:10.1093/cercor/13.10.1051

Magri C, Whittingstall K, Singh V, Logothetis NK, Panzeri S. 2009. A toolbox for the fast information analysis of multiple-site LFP, EEG and spike train recordings. $B M C$ neuroscience 10:81. doi:10.1186/1471-2202-10-81

Maheswaranathan N, Baccus SA, Ganguli S. 2018. Inferring hidden structure in multilayered neural circuits. PLoS Computational Biology 18:e1006291. doi:doi.org/10.1101/120956

Margrie TW, Brecht M, Sakmann B. 2002. In vivo, low-resistance, whole-cell recordings from neurons in the anaesthetized and awake mammalian brain. Pflugers Arch 444:491-498. doi:10.1007/s00424-002-0831-z

Marre O, Botella-Soler V, Simmons KD, Mora T, Tkačik G, Berry MJ. 2015. High Accuracy Decoding of Dynamical Motion from a Large Retinal Population. PLoS Computational Biology 11:1-25. doi:10.1371/journal.pcbi.1004304

Martens MB, Celikel T, Tiesinga PHE. 2015. A Developmental Switch for Hebbian Plasticity. PLOS Computational Biology 11:e1004386. doi:10.1371/journal.pcbi.1004386

Mastrogiuseppe F, Ostojic S. 2017. Linking connectivity, dynamics and computations in recurrent neural networks. arXiv 1711.09672. doi:10.1016/j.neuron.2018.07.003

Narayanan RT, Egger R, Johnson AS, Mansvelder HD, Sakmann B, de Kock CPJ, Oberlaender M. 2015. Beyond Columnar Organization: Cell Type- and Target Layer-Specific Principles of Horizontal Axon Projection Patterns in Rat Vibrissal Cortex. Cereb Cortex 25:4450-4468. doi:10.1093/cercor/bhv053

Nemenman I, Bialek W, de Ruyter van Steveninck R. 2004. Entropy and information in neural spike trains: Progress on the sampling problem. Phys Rev E 69:056111. doi:10.1103/PhysRevE.69.056111

Oberlaender M, de Kock CPJ, Bruno RM, Ramirez A, Meyer HS, Dercksen VJ, Helmstaedter M, Sakmann B. 2012. Cell Type-Specific Three-Dimensional Structure of Thalamocortical Circuits in a Column of Rat Vibrissal Cortex. Cereb Cortex 22:2375-2391. doi:10.1093/cercor/bhr317

Panzeri S, Brunel N, Logothetis NK, Kayser C. 2010. Sensory neural codes using multiplexed temporal scales. Trends in Neurosciences 33:111-120. doi:10.1016/j.tins.2009.12.001

Panzeri S, Diamond ME. 2010. Information carried by population spike times in the whisker sensory cortex can be decoded without knowledge of stimulus time. Front Synaptic Neurosci 2. doi:10.3389/fnsyn.2010.00017

Panzeri S, Petersen RS, Schultz SR, Lebedev M, Diamond ME. 2001. The role of spike timing in the coding of stimulus location in rat somatosensory cortex. Neuron 29:769-77.

Panzeri S, Treves A. 1996. Analytical estimates of limited sampling biases in different 
1023

1024

1025

1026

1027

1028

1029

1030

1031

1032

1033

1034

1035

1036

1037

1038

1039

1040

1041

1042

1043

1044

1045

1046

1047

1048

1049

1050

1051

1052

1053

1054

1055

1056

1057

1058

1059

1060

1061 information measures. Network: Computation in Neural Systems 7:87-107. doi:10.1080/0954898X.1996.11978656

Peron SP, Freeman J, Iyer V, Guo C, Svoboda K. 2015. A Cellular Resolution Map of Barrel Cortex Activity during Tactile Behavior. Neuron 86:783-799. doi:10.1016/j.neuron.2015.03.027

Petersen CCH. 2019. Sensorimotor processing in the rodent barrel cortex. Nature Reviews Neuroscience 20:533-546. doi:10.1038/s41583-019-0200-y

Petersen RS, Panzeri S, Diamond ME. 2002. Population coding in somatosensory cortex. Current Opinion in Neurobiology 12:441-447.

Petersen RS, Panzeri S, Diamond ME. 2001. Population coding of stimulus location in rat somatosensory cortex. Neuron 32:503-14.

Poulet JFA, Petersen CCH. 2008. Internal brain state regulates membrane potential synchrony in barrel cortex of behaving mice. Nature 454:881-885. doi:10.1038/nature07150

Proville RD, Spolidoro M, Guyon N, Dugué GP, Selimi F, Isope P, Popa D, Léna C. 2014. Cerebellum involvement in cortical sensorimotor circuits for the control of voluntary movements. Nature Neuroscience 17:1233-1239. doi:10.1038/nn.3773

Quian Quiroga R, Panzeri S. 2009. Extracting information from neuronal populations: information theory and decoding approaches. Nature reviews Neuroscience 10:173-85. doi:10.1038/nrn2578

Renart A, Rocha JD, Barthó P, Hollender L, Parga N, Reyes A, Harris KD. 2010. The Asynchronous State in Cortical Circuits. Science 327:587-590. doi:10.1126/science.1179850.The

Rosenblatt F. 1958. The perceptron: A probabilistic model for information storage and organization in the brain. Psychological Review 65:386-408.

Seung HS, Yuste R. 2012. Neural Networks In: Kandel ER, Schwartz JH, Jessell TM, Siegelbaum SA, Hudspeth AJ, editors. Principles of Neural Science. McGraw-Hill. pp. 1581-1600.

Sriram B, Li L, Cruz-Martín A, Ghosh A. 2020. A Sparse Probabilistic Code Underlies the Limits of Behavioral Discrimination. Cereb Cortex 30:1040-1055. doi:10.1093/cercor/bhz147

Staiger JF, Bojak I, Miceli S, Schubert D. 2015. A gradual depth-dependent change in connectivity features of supragranular pyramidal cells in rat barrel cortex. Brain Struct Funct 220:1317-1337. doi:10.1007/s00429-014-0726-8

Stewart RS, Huang C, Arnett MT, Celikel T. 2013. Spontaneous oscillations in intrinsic signals reveal the structure of cerebral vasculature. Journal of Neurophysiology 109:3094-3104. doi:10.1152/jn.01200.2011

Stüttgen MC, Schwarz C. 2010. Integration of Vibrotactile Signals for Whisker-Related Perception in Rats Is Governed by Short Time Constants: Comparison of Neurometric and Psychometric Detection Performance. J Neurosci 30:2060-2069. 
1062

1063

1064

1065

1066

1067

1068

1069

1070

1071

1072

1073

1074

1075

1076

1077

1078

1079

1080

1081

doi:10.1523/JNEUROSCI.3943-09.2010

Thomson AM, Lamy C. 2007. Functional maps of neocortical local circuitry. Front Neurosci 1. doi:10.3389/neuro.01.1.1.002.2007

Ujfalussy BB, Makara JK, Branco T, Lengyel M. 2015. Dendritic nonlinearities are tuned for efficient spike-based computations in cortical circuits. eLife 4:e10056. doi:10.7554/eLife.10056

Victor JD. 2009. Approaches to Information-Theoretic Analysis of Neural Activity. Biological Theory 1:302-316.

Voigts J, Herman DH, Celikel T. 2015. Tactile Object Localization by Anticipatory Whisker Motion. Journal of neurophysiology 113:620-632. doi:10.1152/jn.00241.2014

Voigts J, Sakmann B, Celikel T. 2008. Unsupervised whisker tracking in unrestrained behaving animals. Journal of neurophysiology 100:504-515. doi:10.1152/jn.00012.2008.

Weinberg RJ. 1997. Are Topographic Maps Fundamental to Sensory Processing ? Brain Research Bulletin 44:113-116. doi:10.1016/S0361-9230(97)00094-4

Wilent WB, Contreras D. 2004. Synaptic Responses to Whisker Deflections in Rat Barrel Cortex as a Function of Cortical Layer and Stimulus Intensity. Journal of Neuroscience 24:3985-3998. doi:10.1523/JNEUROSCI.5782-03.2004

Zeldenrust F, Calcini N, Yan X, Bijlsma A, Celikel T. 2020. Cell type specific information transfer for sparse coding. bioRxiv 2020.11.06.371658. doi:10.1101/2020.11.06.371658 